TRANSACTIONS OF THE

AMERICAN MATHEMATICAL SOCIETY

Volume 364, Number 3, March 2012, Pages 1369-1393

S 0002-9947(2011)05368-7

Article electronically published on September 2, 2011

\title{
FINITE WEYL GROUPOIDS OF RANK THREE
}

\author{
M. CUNTZ AND I. HECKENBERGER
}

\begin{abstract}
We continue our study of Cartan schemes and their Weyl groupoids and obtain a complete list of all connected simply connected Cartan schemes of rank three for which the real roots form a finite irreducible root system. We achieve this result by providing an algorithm which determines all the root systems and eventually terminates: Up to equivalence there are exactly 55 such Cartan schemes, and the number of corresponding real roots varies between 6 and 37. We identify those Weyl groupoids which appear in the classification of Nichols algebras of diagonal type.
\end{abstract}

\section{INTRODUCTION}

Root systems associated with Cartan matrices are widely studied structures in many areas of mathematics; see Bou68 for the fundamentals. The origins of the theory of root systems go back at least to the study of Lie groups by Lie, Killing and Cartan. The symmetry of the root system is commonly known as its Weyl group. Root systems associated with a family of Cartan matrices appeared first in connection with Lie superalgebras Kac77, Prop. 2.5.6] and with Nichols algebras Hec06, Hec08. The corresponding symmetry is not a group but a groupoid, and is called the Weyl groupoid of the root system.

Weyl groupoids of root systems properly generalize Weyl groups. The nice properties of this more general structure have been the main motivation to develop an axiomatic approach to the theory; see [HY08, CH09c]. In particular, Weyl groupoids are generated by reflections and Coxeter relations, and they satisfy a Matsumoto type theorem [HY08. To more clearly see the extent of generality it would be desirable to have a classification of finite Weyl groupoids 11 However, already the appearance of a large family of examples of Lie superalgebras and Nichols algebras of diagonal type indicated that a classification of finite Weyl groupoids is probably much more complicated than the classification of finite Weyl groups. Additionally, many of the usual classification tools are not available in this context because of the lack of the adjoint action and a positive definite bilinear form.

Received by the editors December 4, 2009 and, in revised form, March 22, 2010, March 25, 2010 and April 7, 2010.

2010 Mathematics Subject Classification. Primary 20F55, 16T30, 52C30.

The second author was supported by the German Research Foundation (DFG) via a Heisenberg fellowship.

${ }^{1}$ In this introduction by a Weyl groupoid we will mean the Weyl groupoid of a connected Cartan scheme, and we assume that the real roots associated to the Weyl groupoid form an irreducible root system in the sense of [CH09c]. Further, our sloppy but meaningful terminology of classification of finite Weyl groupoids should be understood as the classification of connected simply connected Cartan schemes such that this root system is finite.

(C)2011 American Mathematical Society 
In previous work (see [CH09b and $\mathrm{CH} 09 \mathrm{a}]$ ) we have been able to determine all finite Weyl groupoids of rank two. The result of this classification is surprisingly nice: We found a close relationship to the theory of continued fractions and to cluster algebras of type $A$. The structure of finite rank two Weyl groupoids and the associated root systems has a natural characterization in terms of triangulations of convex polygons by nonintersecting diagonals. In particular, there are infinitely many such groupoids.

At first view there is no reason to assume that the situation for finite Weyl groupoids of rank three would be much different from the rank two case. In this paper we first give some theoretical indications which strengthen the opposite point of view. For example in Theorem 3.13 we show that the entries of the Cartan matrices in a finite Weyl groupoid cannot be smaller than -7 . Recall that for Weyl groupoids there is no lower bound for the possible entries of generalized Cartan matrices. Our main achievement in this paper is then to provide an algorithm to classify finite Weyl groupoids of rank three. Our algorithm terminates within a short time, and produces a complete list of representatives of root systems. In the appendix we list the root systems characterizing the Weyl groupoids of the classification: There are 55 of them which correspond to pairwise nonisomorphic Weyl groupoids. The number of positive roots in these root systems varies between 6 and 37. Among our root systems are the usual root systems of types $A_{3}, B_{3}$, and $C_{3}$, but for most of the other examples we don't yet have an explanation.

It is remarkable that the number 37 has a particular meaning for simplicial arrangements in the real projective plane. An arrangement is the complex generated by a family of straight lines not forming a pencil. The vertices of the complex are the intersection points of the lines, the edges are the segments of the lines between two vertices, and the faces are the connected components of the complement of the set of lines generating the arrangement. An arrangement is called simplicial if all faces are triangles. Simplicial arrangements have been introduced in Mel41. The classification of simplicial arrangements in the real projective plane is an open problem. The largest known exceptional example is generated by 37 lines. Grünbaum conjectures that the list given in Grü09 is complete. In our appendix we provide some data of our root systems which can be used to compare Grünbaum's list with Weyl groupoids. There is an astonishing analogy between the two lists, but more work has to be done to be able to explain the precise relationship. This would be desirable in particular since our classification of finite Weyl groupoids of rank three does not give any evidence for the range of solutions besides the explicit computer calculation.

In order to ensure the termination of our algorithm, besides Theorem 3.13 we use a weak convexity property of certain affine hyperplanes; see Theorem 3.11 We can show that any positive root in an affine hyperplane "next to the origin" is either simple or can be written as the sum of a simple root and another positive root. Our algorithm finally becomes practicable by the use of Proposition 3.6, which can be interpreted as another weak convexity property for affine hyperplanes. It is hard to say which of these theorems is the most valuable because avoiding any of them makes the algorithm impracticable (unless one has some replacement).

It should also be mentioned that using the knowledge on finite Weyl groupoids of rank three and an enhanced version of the algorithm, it is now possible to push the classification up to rank seven. It is thus conceivable that with some additional theory a complete classification will be within reach. 
The paper is organized as follows. We start with two sections proving the necessary theorems to formulate the algorithm: the results which do not require that the rank is three are in Section 2 , the obstructions for rank three are in Section 3. We then describe the algorithm in the next section. Finally we summarize the resulting data and make some observations in the last section.

\section{Cartan schemes and Weyl groupoids}

We mainly follow the notation in $\mathrm{CH} 09 \mathrm{c}, \mathrm{CH} 09 \mathrm{~b}]$. The fundamentals of the general theory have been developed in [HY08] using a somewhat different terminology. Let us start by recalling the main definitions.

Let $I$ be a nonempty finite set and $\left\{\alpha_{i} \mid i \in I\right\}$ the standard basis of $\mathbb{Z}^{I}$. By [Kac90, §1.1] a generalized Cartan matrix $C=\left(c_{i j}\right)_{i, j \in I}$ is a matrix in $\mathbb{Z}^{I \times I}$ such that

(M1) $c_{i i}=2$ and $c_{j k} \leq 0$ for all $i, j, k \in I$ with $j \neq k$,

(M2) if $i, j \in I$ and $c_{i j}=0$, then $c_{j i}=0$.

Let $A$ be a nonempty set, $\rho_{i}: A \rightarrow A$ a map for all $i \in I$, and $C^{a}=\left(c_{j k}^{a}\right)_{j, k \in I}$ a generalized Cartan matrix in $\mathbb{Z}^{I \times I}$ for all $a \in A$. The quadruple

$$
\mathcal{C}=\mathcal{C}\left(I, A,\left(\rho_{i}\right)_{i \in I},\left(C^{a}\right)_{a \in A}\right)
$$

is called a Cartan scheme if

(C1) $\rho_{i}^{2}=$ id for all $i \in I$,

(C2) $c_{i j}^{a}=c_{i j}^{\rho_{i}(a)}$ for all $a \in A$ and $i, j \in I$.

Let $\mathcal{C}=\mathcal{C}\left(I, A,\left(\rho_{i}\right)_{i \in I},\left(C^{a}\right)_{a \in A}\right)$ be a Cartan scheme. For all $i \in I$ and $a \in A$ define $\sigma_{i}^{a} \in \operatorname{Aut}\left(\mathbb{Z}^{I}\right)$ by

$$
\sigma_{i}^{a}\left(\alpha_{j}\right)=\alpha_{j}-c_{i j}^{a} \alpha_{i} \quad \text { for all } j \in I .
$$

The Weyl groupoid of $\mathcal{C}$ is the category $\mathcal{W}(\mathcal{C})$ such that $\operatorname{Ob}(\mathcal{W}(\mathcal{C}))=A$, and the morphisms are compositions of maps $\sigma_{i}^{a}$ with $i \in I$ and $a \in A$, where $\sigma_{i}^{a}$ is considered as an element in $\operatorname{Hom}\left(a, \rho_{i}(a)\right)$. The category $\mathcal{W}(\mathcal{C})$ is a groupoid in the sense that all morphisms are isomorphisms. The set of morphisms of $\mathcal{W}(\mathcal{C})$ is denoted by $\operatorname{Hom}(\mathcal{W}(\mathcal{C}))$, and we use the notation

$$
\operatorname{Hom}(a, \mathcal{W}(\mathcal{C}))=\bigcup_{b \in A} \operatorname{Hom}(a, b) \quad \text { (disjoint union) }
$$

For notational convenience we will often neglect upper indices referring to elements of $A$ if they are uniquely determined by the context. For example, the morphism $\sigma_{i_{1}}^{\rho_{i_{2}} \cdots \rho_{i_{k}}(a)} \cdots \sigma_{i_{k-1}}^{\rho_{i_{k}(a)}} \sigma_{i_{k}}^{a} \in \operatorname{Hom}(a, b)$, where $k \in \mathbb{N}, i_{1}, \ldots, i_{k} \in I$, and $b=\rho_{i_{1}} \cdots \rho_{i_{k}}(a)$, will be denoted by $\sigma_{i_{1}} \cdots \sigma_{i_{k}}^{a}$ or by $\operatorname{id}_{b} \sigma_{i_{1}} \cdots \sigma_{i_{k}}$. The cardinality of $I$ is termed the rank of $\mathcal{W}(\mathcal{C})$. A Cartan scheme is called connected if its Weyl groupoid is connected, that is, if for all $a, b \in A$ there exists $w \in \operatorname{Hom}(a, b)$. The Cartan scheme is called simply connected if $\operatorname{Hom}(a, a)=\left\{\operatorname{id}_{a}\right\}$ for all $a \in A$.

Let $\mathcal{C}$ be a Cartan scheme. For all $a \in A$ let

$$
\left(R^{\mathrm{re}}\right)^{a}=\left\{\operatorname{id}_{a} \sigma_{i_{1}} \cdots \sigma_{i_{k}}\left(\alpha_{j}\right) \mid k \in \mathbb{N}_{0}, i_{1}, \ldots, i_{k}, j \in I\right\} \subset \mathbb{Z}^{I} .
$$

The elements of the set $\left(R^{\mathrm{re}}\right)^{a}$ are called real roots (at $\left.a\right)$. The pair $\left(\mathcal{C},\left(\left(R^{\mathrm{re}}\right)^{a}\right)_{a \in A}\right)$ is denoted by $\mathcal{R}^{\mathrm{re}}(\mathcal{C})$. A real root $\alpha \in\left(R^{\mathrm{re}}\right)^{a}$, where $a \in A$, is called positive (resp. negative) if $\alpha \in \mathbb{N}_{0}^{I}$ (resp. $\alpha \in-\mathbb{N}_{0}^{I}$ ). In contrast to real roots associated to a single generalized Cartan matrix, $\left(R^{\mathrm{re}}\right)^{a}$ may contain elements which are neither positive 


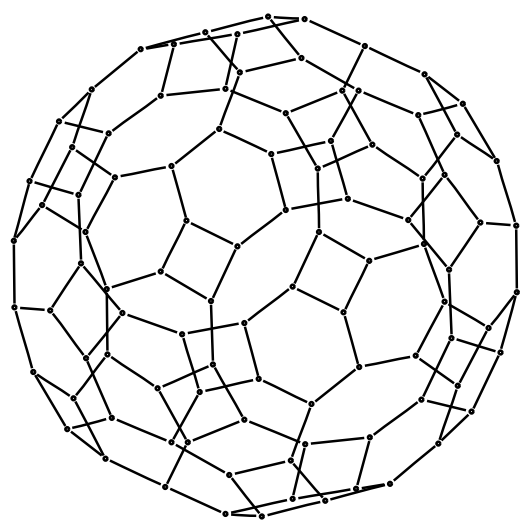

Figure 1. The object change diagram of a Cartan scheme of rank three (nr. 15 in Table 1$)$

nor negative. A good general theory, which is relevant for example for the study of Nichols algebras, can be obtained if $\left(R^{\mathrm{re}}\right)^{a}$ satisfies additional properties.

Let $\mathcal{C}=\mathcal{C}\left(I, A,\left(\rho_{i}\right)_{i \in I},\left(C^{a}\right)_{a \in A}\right)$ be a Cartan scheme. For all $a \in A$, let $R^{a} \subset \mathbb{Z}^{I}$, and define $m_{i, j}^{a}=\left|R^{a} \cap\left(\mathbb{N}_{0} \alpha_{i}+\mathbb{N}_{0} \alpha_{j}\right)\right|$ for all $i, j \in I$ and $a \in A$. We say that

$$
\mathcal{R}=\mathcal{R}\left(\mathcal{C},\left(R^{a}\right)_{a \in A}\right)
$$

is a root system of type $\mathcal{C}$ if it satisfies the following axioms.

(R1) $R^{a}=R_{+}^{a} \cup-R_{+}^{a}$, where $R_{+}^{a}=R^{a} \cap \mathbb{N}_{0}^{I}$, for all $a \in A$.

(R2) $R^{a} \cap \mathbb{Z} \alpha_{i}=\left\{\alpha_{i},-\alpha_{i}\right\}$ for all $i \in I, a \in A$.

(R3) $\sigma_{i}^{a}\left(R^{a}\right)=R^{\rho_{i}(a)}$ for all $i \in I, a \in A$.

(R4) If $i, j \in I$ and $a \in A$ such that $i \neq j$ and $m_{i, j}^{a}$ is finite, then $\left(\rho_{i} \rho_{j}\right)^{m_{i, j}^{a}(a)=}$ a.

The axioms (R2) and (R3) are always fulfilled for $\mathcal{R}^{\text {re }}$. The root system $\mathcal{R}$ is called finite if for all $a \in A$ the set $R^{a}$ is finite. By [CH09c, Prop. 2.12], if $\mathcal{R}$ is a finite root system of type $\mathcal{C}$, then $\mathcal{R}=\mathcal{R}^{\text {re }}$, and hence $\mathcal{R}^{\text {re }}$ is a root system of type $\mathcal{C}$ in that case.

In [CH09c, Def. 4.3] the concept of an irreducible root system of type $\mathcal{C}$ was defined. By [CH09c, Prop.4.6], if $\mathcal{C}$ is a Cartan scheme and $\mathcal{R}$ is a finite root system of type $\mathcal{C}$, then $\mathcal{R}$ is irreducible if and only if for all $a \in A$ the generalized Cartan matrix $C^{a}$ is indecomposable. If $\mathcal{C}$ is also connected, then it suffices to require that there exists $a \in A$ such that $C^{a}$ is indecomposable.

Let $\mathcal{C}=\mathcal{C}\left(I, A,\left(\rho_{i}\right)_{i \in I},\left(C^{a}\right)_{a \in A}\right)$ be a Cartan scheme. Let $\Gamma$ be a nondirected graph, such that the vertices of $\Gamma$ correspond to the elements of $A$. Assume that for all $i \in I$ and $a \in A$ with $\rho_{i}(a) \neq a$ there is precisely one edge between the vertices $a$ and $\rho_{i}(a)$ with label $i$, and all edges of $\Gamma$ are given in this way. The graph $\Gamma$ is called the object change diagram of $\mathcal{C}$.

In the rest of this section, let $\mathcal{C}=\mathcal{C}\left(I, A,\left(\rho_{i}\right)_{i \in I},\left(C^{a}\right)_{a \in A}\right)$ be a Cartan scheme such that $\mathcal{R}^{\mathrm{re}}(\mathcal{C})$ is a finite root system. For brevity we will write $R^{a}$ instead of $\left(R^{\mathrm{re}}\right)^{a}$ for all $a \in A$. We say that a subgroup $H \subset \mathbb{Z}^{I}$ is a hyperplane if $\mathbb{Z}^{I} / H \cong \mathbb{Z}$. Then $\operatorname{rk} H=\# I-1$ is the rank of $H$. Sometimes we will identify $\mathbb{Z}^{I}$ with its image under the canonical embedding $\mathbb{Z}^{I} \rightarrow \mathbb{Q} \otimes_{\mathbb{Z}} \mathbb{Z}^{I} \cong \mathbb{Q}^{I}$. 
Lemma 2.1. Let $a \in A$ and let $H \subset \mathbb{Z}^{I}$ be a hyperplane. Suppose that $H$ contains rk $H$ linearly independent elements of $R^{a}$. Let $\mathfrak{n}_{H}$ be a normal vector of $H$ in $\mathbb{Q}^{I}$ with respect to a scalar product $(\cdot, \cdot)$ on $\mathbb{Q}^{I}$. If $\left(\mathfrak{n}_{H}, \alpha\right) \geq 0$ for all $\alpha \in R_{+}^{a}$, then $H$ contains $\mathrm{rk} H$ simple roots, and all roots contained in $H$ are linear combinations of these simple roots.

Proof. The assumptions imply that any positive root in $H$ is a linear combination of simple roots contained in $H$. Since $R^{a}=R_{+}^{a} \cup-R_{+}^{a}$, this implies the claim.

Lemma 2.2. Let $a \in A$ and let $H \subset \mathbb{Z}^{I}$ be a hyperplane. Suppose that $H$ contains $\mathrm{rk} H$ linearly independent elements of $R^{a}$. Then there exist $b \in A$ and $w \in \operatorname{Hom}(a, b)$ such that $w(H)$ contains $\mathrm{rk} H$ simple roots.

Proof. Let $(\cdot, \cdot)$ be a scalar product on $\mathbb{Q}^{I}$. Choose a normal vector $\mathfrak{n}_{H}$ of $H$ in $\mathbb{Q}^{I}$ with respect to $(\cdot, \cdot)$. Let $m=\#\left\{\alpha \in R_{+}^{a} \mid\left(\mathfrak{n}_{H}, \alpha\right)<0\right\}$. Since $\mathcal{R}^{\text {re }}(\mathcal{C})$ is finite, $m$ is a nonnegative integer. We proceed by induction on $m$. If $m=0$, then $H$ contains rk $H$ simple roots by Lemma 2.1. Otherwise let $j \in I$ with $\left(\mathfrak{n}_{H}, \alpha_{j}\right)<0$. Let $a^{\prime}=\rho_{j}(a)$ and $H^{\prime}=\sigma_{j}^{a}(H)$. Then $\sigma_{j}^{a}\left(\mathfrak{n}_{H}\right)$ is a normal vector of $H^{\prime}$ with respect to the scalar product $(\cdot, \cdot)^{\prime}=\left(\sigma_{j}^{\rho_{j}(a)}(\cdot), \sigma_{j}^{\rho_{j}(a)}(\cdot)\right)$. Since $\sigma_{j}^{a}: R_{+}^{a} \backslash\left\{\alpha_{j}\right\} \rightarrow R_{+}^{a^{\prime}} \backslash\left\{\alpha_{j}\right\}$ is a bijection and $\sigma_{j}^{a}\left(\alpha_{j}\right)=-\alpha_{j}$, we conclude that

$$
\#\left\{\beta \in R_{+}^{a^{\prime}} \mid\left(\sigma_{j}^{a}\left(\mathfrak{n}_{H}\right), \beta\right)^{\prime}<0\right\}=\#\left\{\alpha \in R_{+}^{a} \mid\left(\mathfrak{n}_{H}, \alpha\right)<0\right\}-1 .
$$

By the induction hypothesis there exists $b \in A$ and $w^{\prime} \in \operatorname{Hom}\left(a^{\prime}, b\right)$ such that $w^{\prime}\left(H^{\prime}\right)$ contains $\mathrm{rk} H^{\prime}=\mathrm{rk} H$ simple roots. Then the claim of the lemma holds for $w=w^{\prime} \sigma_{j}^{a}$.

The following "volume" functions will be useful for our analysis. Let $k \in \mathbb{N}$ with $k \leq \# I$. By the Smith normal form there is a unique left $\operatorname{GL}\left(\mathbb{Z}^{I}\right)$-invariant right $\mathrm{GL}\left(\mathbb{Z}^{k}\right)$-invariant function $\operatorname{Vol}_{k}:\left(\mathbb{Z}^{I}\right)^{k} \rightarrow \mathbb{Z}$ such that

$$
\operatorname{Vol}_{k}\left(a_{1} \alpha_{1}, \ldots, a_{k} \alpha_{k}\right)=\left|a_{1} \cdots a_{k}\right| \text { for all } a_{1}, \ldots, a_{k} \in \mathbb{Z},
$$

where $|\cdot|$ denotes absolute value. In particular, if $k=1$ and $\beta \in \mathbb{Z}^{I} \backslash\{0\}$, then $\operatorname{Vol}_{1}(\beta)$ is the largest integer $v$ such that $\beta=v \beta^{\prime}$ for some $\beta^{\prime} \in \mathbb{Z}^{I}$. Further, if $k=\# I$ and $\beta_{1}, \ldots, \beta_{k} \in \mathbb{Z}^{I}$, then $\operatorname{Vol}_{k}\left(\beta_{1}, \ldots, \beta_{k}\right)$ is the absolute value of the determinant of the matrix with columns $\beta_{1}, \ldots, \beta_{k}$.

Let $a \in A, k \in\{1,2, \ldots, \# I\}$, and let $\beta_{1}, \ldots, \beta_{k} \in R^{a}$ be linearly independent elements. We write $V^{a}\left(\beta_{1}, \ldots, \beta_{k}\right)$ for the unique maximal subgroup $V \subseteq \mathbb{Z}^{I}$ of rank $k$ which contains $\beta_{1}, \ldots, \beta_{k}$. Then $\mathbb{Z}^{I} / V^{a}\left(\beta_{1}, \ldots, \beta_{k}\right)$ is free. In particular, $V^{a}\left(\beta_{1}, \ldots, \beta_{\# I}\right)=\mathbb{Z}^{I}$ for all $a \in A$ and any linearly independent subset $\left\{\beta_{1}, \ldots, \beta_{\# I}\right\}$ of $R^{a}$.

Definition 2.3. Let $W \subseteq \mathbb{Z}^{I}$ be a cofree subgroup (that is, $\mathbb{Z}^{I} / W$ is free) of rank $k$. We say that $\left\{\beta_{1}, \ldots, \beta_{k}\right\}$ is a base for $W$ at $a$ if $\beta_{i} \in W$ for all $i \in\{1, \ldots, k\}$ and $W \cap R^{a} \subseteq \sum_{i=1}^{k} \mathbb{N}_{0} \beta_{i} \cup-\sum_{i=1}^{k} \mathbb{N}_{0} \beta_{i}$.

Now we discuss the relationship of linearly independent roots in a root system. Recall that $\mathcal{C}$ is a Cartan scheme such that $\mathcal{R}^{\text {re }}(\mathcal{C})$ is a finite root system of type $\mathcal{C}$.

Theorem 2.4. Let $a \in A, k \in\{1, \ldots, \# I\}$, and let $\beta_{1}, \ldots, \beta_{k} \in R^{a}$ be linearly independent roots. Then there exist $b \in A, w \in \operatorname{Hom}(a, b)$, and a permutation $\tau$ of 
I such that

$$
w\left(\beta_{i}\right) \in \operatorname{span}_{\mathbb{Z}}\left\{\alpha_{\tau(1)}, \ldots, \alpha_{\tau(i)}\right\} \cap R_{+}^{b}
$$

for all $i \in\{1, \ldots, k\}$.

Proof. Let $r=\# I$. Since $R^{a}$ contains $r$ simple roots, any linearly independent subset of $R^{a}$ can be enlarged to a linearly independent subset of $r$ elements. Hence it suffices to prove the theorem for $k=r$. We proceed by induction on $r$. If $r=1$, then the claim holds.

Assume that $r>1$. Lemma 2.2 with $H=V^{a}\left(\beta_{1}, \ldots, \beta_{r-1}\right)$ tells us that there exist $d \in A$ and $v \in \operatorname{Hom}(a, d)$ such that $v(H)$ is spanned by simple roots. By multiplying $v$ from the left with the longest element of $\mathcal{W}(\mathcal{C})$ in the case that $v\left(\beta_{r}\right) \in-\mathbb{N}_{0}^{I}$, we may even assume that $v\left(\beta_{r}\right) \in \mathbb{N}_{0}^{I}$. Now let $J$ be the subset of $I$ such that $\# J=r-1$ and $\alpha_{i} \in v(H)$ for all $i \in J$. Consider the restriction $\left.\mathcal{R}^{\mathrm{re}}(\mathcal{C})\right|_{J}$ of $\mathcal{R}^{\mathrm{re}}(\mathcal{C})$ to the index set $J$; see [CH09c, Def. 4.1]. Since $v\left(\beta_{i}\right) \in H$ for all $i \in\{1, \ldots, r-1\}$, the induction hypothesis provides us with $b \in A, u \in \operatorname{Hom}(d, b)$, and a permutation $\tau^{\prime}$ of $J$ such that $u$ is a product of simple reflections $\sigma_{i}$, where $i \in J$, and

$$
u v\left(\beta_{n}\right) \in \operatorname{span}_{\mathbb{Z}}\left\{\alpha_{\tau^{\prime}\left(j_{1}\right)}, \ldots, \alpha_{\tau^{\prime}\left(j_{n}\right)}\right\} \cap R_{+}^{b}
$$

for all $n \in\{1,2, \ldots, r-1\}$, where $J=\left\{j_{1}, \ldots, j_{r-1}\right\}$. Since $v\left(\beta_{r}\right) \notin v(H)$ and $v\left(\beta_{r}\right) \in \mathbb{N}_{0}^{I}$, the $i$-th entry of $v\left(\beta_{r}\right)$, where $i \in I \backslash J$, is positive. This entry does not change if we apply $u$. Therefore $u v\left(\beta_{r}\right) \in \mathbb{N}_{0}^{I}$, and hence the theorem holds with $w=u v \in \operatorname{Hom}(a, b)$ and with $\tau$ the unique permutation with $\tau(n)=\tau^{\prime}\left(j_{n}\right)$ for all $n \in\{1, \ldots, r-1\}$.

Corollary 2.5. Let $a \in A, k \in\{1, \ldots, \# I\}$, and let $\beta_{1}, \ldots, \beta_{k} \in R^{a}$ be linearly independent elements. Then $\left\{\beta_{1}, \ldots, \beta_{k}\right\}$ is a base for $V^{a}\left(\beta_{1}, \ldots, \beta_{k}\right)$ at $a$ if and only if there exist $b \in A, w \in \operatorname{Hom}(a, b)$, and a permutation $\tau$ of $I$ such that $w\left(\beta_{i}\right)=\alpha_{\tau(i)}$ for all $i \in\{1, \ldots, k\}$. In this case $\operatorname{Vol}_{k}\left(\beta_{1}, \ldots, \beta_{k}\right)=1$.

Proof. The if part of the claim holds by definition of a base and by the axioms for root systems.

Let $b, w$ and $\tau$ be as in Theorem 2.4. Let $i \in\{1, \ldots, k\}$. The elements $w\left(\beta_{1}\right), \ldots$, $w\left(\beta_{i}\right)$ are linearly independent and are contained in $V^{b}\left(\alpha_{\tau(1)}, \ldots, \alpha_{\tau(i)}\right)$. Thus $\alpha_{\tau(i)}$ is a rational linear combination of $w\left(\beta_{1}\right), \ldots, w\left(\beta_{i}\right)$. Now by assumption, $\left\{w\left(\beta_{1}\right), \ldots, w\left(\beta_{k}\right)\right\}$ is a base for $V^{b}\left(w\left(\beta_{1}\right), \ldots, w\left(\beta_{k}\right)\right)$ at $b$. Hence $\alpha_{\tau(i)}$ is a linear combination of the positive roots $w\left(\beta_{1}\right), \ldots, w\left(\beta_{i}\right)$ with nonnegative integer coefficients. This is possible only if $\left\{w\left(\beta_{1}\right), \ldots, w\left(\beta_{i}\right)\right\}$ contains $\alpha_{\tau(i)}$. By induction on $i$ we obtain that $\alpha_{\tau(i)}=w\left(\beta_{i}\right)$.

In the special case $k=\# I$ the above corollary tells us that the bases of $\mathbb{Z}^{I}$ at an object $a \in A$ are precisely those subsets which can be obtained as the image, up to a permutation, of the standard basis of $\mathbb{Z}^{I}$ under the action of an element of $\mathcal{W}(\mathcal{C})$.

In $\mathrm{CH} 09 \mathrm{a}$ the notion of an $\mathcal{F}$-sequence was given, and it was used to explain the structure of root systems of rank two. Consider on $\mathbb{N}_{0}^{2}$ the total ordering $\leq_{\mathbb{Q}}$, where $\left(a_{1}, a_{2}\right) \leq_{\mathbb{Q}}\left(b_{1}, b_{2}\right)$ if and only if $a_{1} b_{2} \leq a_{2} b_{1}$. A finite sequence $\left(v_{1}, \ldots, v_{n}\right)$ of vectors in $\mathbb{N}_{0}^{2}$ is an $\mathcal{F}$-sequence if and only if $v_{1}<\mathbb{Q} v_{2}<\mathbb{Q} \cdots<_{\mathbb{Q}} v_{n}$ and one of 
the following holds:

- $n=2, v_{1}=(0,1)$, and $v_{2}=(1,0)$;

- $n>2$ and there exists $i \in\{2,3, \ldots, n-1\}$ such that $v_{i}=v_{i-1}+v_{i+1}$ and $\left(v_{1}, \ldots, v_{i-1} \cdot v_{i+1}, \ldots, v_{n}\right)$ is an $\mathcal{F}$-sequence.

In particular, any $\mathcal{F}$-sequence of length $\geq 3$ contains $(1,1)$.

Proposition 2.6 ([CH09a, Prop. 3.7]). Let $\mathcal{C}$ be a Cartan scheme of rank two. Assume that $\mathcal{R}^{\mathrm{re}}(\mathcal{C})$ is a finite root system. Then for any $a \in A$ the set $R_{+}^{a}$ ordered by $\leq_{\mathbb{Q}}$ is an $\mathcal{F}$-sequence.

Proposition 2.7 ([CH09a, Cor. 3.8]). Let $\mathcal{C}$ be a Cartan scheme of rank two. Assume that $\mathcal{R}^{\mathrm{re}}(\mathcal{C})$ is a finite root system. Let $a \in A$ and let $\beta \in R_{+}^{a}$. Then either $\beta$ is simple or it is the sum of two positive roots.

Corollary 2.8. Let $a \in A, n \in \mathbb{N}$, and let $\alpha, \beta \in R^{a}$ such that $\beta-n \alpha \in R^{a}$. Assume that $\{\alpha, \beta-n \alpha\}$ is a base for $V^{a}(\alpha, \beta)$ at a. Then $\beta-k \alpha \in R^{a}$ for all $k \in\{1,2, \ldots, n\}$.

Proof. By Corollary 2.5 there exist $b \in A, w \in \operatorname{Hom}(a, b)$, and $i, j \in I$ such that $w(\alpha)=\alpha_{i}, w(\beta-n \alpha)=\alpha_{j}$. Then $n \alpha_{i}+\alpha_{j}=w(\beta) \in R_{+}^{b}$. Hence $(n-k) \alpha_{i}+\alpha_{j} \in R^{b}$ for all $k \in\{1,2, \ldots, n\}$ by Proposition 2.7 and (R2). This yields the claim of the corollary.

Corollary 2.9. Let $a \in A, k \in \mathbb{Z}$, and $i, j \in I$ such that $i \neq j$. Then $\alpha_{j}+k \alpha_{i} \in R^{a}$ if and only if $0 \leq k \leq-c_{i j}^{a}$,

Proof. Axiom (R1) tells us that $\alpha_{j}+k \alpha_{i} \notin R^{a}$ if $k<0$. Since $c_{i j}^{\rho_{i}(a)}=c_{i j}^{a}$ by (C2), Axiom (R3) gives that $\alpha_{j}-c_{i j}^{a} \alpha_{i}=\sigma_{i}^{\rho_{i}(a)}\left(\alpha_{j}\right) \in R^{a}$ and that $\alpha_{j}+k \alpha_{i} \notin R^{a}$ if $k>-c_{i j}^{a}$. Finally, if $0<k<-c_{i j}^{a}$, then $\alpha_{j}+k \alpha_{i} \in R^{a}$ by Corollary 2.8 for $\alpha=\alpha_{i}$, $\beta=\alpha_{j}-c_{i j}^{a} \alpha_{i}$, and $n=-c_{i j}^{a}$.

Proposition 2.7 implies another important fact.

Theorem 2.10. Let $\mathcal{C}$ be a Cartan scheme. Assume that $\mathcal{R}^{\mathrm{re}}(\mathcal{C})$ is a finite root system of type $\mathcal{C}$. Let $a \in A$ and $\alpha \in R_{+}^{a}$. Then either $\alpha$ is simple, or it is the sum of two positive roots.

Proof. Assume that $\alpha$ is not simple. Let $i \in I, b \in A$, and $w \in \operatorname{Hom}(b, a) \operatorname{such}$ that $\alpha=w\left(\alpha_{i}\right)$. Then $\ell(w)>0$. We may assume that for all $j \in I, b^{\prime} \in A$, and $w^{\prime} \in \operatorname{Hom}\left(b^{\prime}, a\right)$ with $w^{\prime}\left(\alpha_{j}\right)=\alpha$ we have $\ell\left(w^{\prime}\right) \geq \ell(w)$. Since $w\left(\alpha_{i}\right) \in \mathbb{N}_{0}^{I}$, we obtain that $\ell\left(w \sigma_{i}\right)>\ell(w)$ [HY08, Cor. 3]. Therefore, there is a $j \in I \backslash\{i\}$ with $\ell\left(w \sigma_{j}\right)<\ell(w)$. Let $w=w_{1} w_{2}$ such that $\ell(w)=\ell\left(w_{1}\right)+\ell\left(w_{2}\right), \ell\left(w_{1}\right)$ minimal and $w_{2}=\ldots \sigma_{i} \sigma_{j} \sigma_{i} \sigma_{j}^{b}$. Assume that $w_{2}=\sigma_{i} \cdots \sigma_{i} \sigma_{j}^{b}$ - the case $w_{2}=\sigma_{j} \cdots \sigma_{i} \sigma_{j}^{b}$ can be treated similarly. The length of $w_{1}$ is minimal, thus $\ell\left(w_{1} \sigma_{j}\right)>\ell\left(w_{1}\right)$, and $\ell(w)=\ell\left(w_{1}\right)+\ell\left(w_{2}\right)$ yields that $\ell\left(w_{1} \sigma_{i}\right)>\ell\left(w_{1}\right)$. Using [HY08, Cor. 3] once more we conclude that

$$
w_{1}\left(\alpha_{i}\right) \in \mathbb{N}_{0}^{I}, \quad w_{1}\left(\alpha_{j}\right) \in \mathbb{N}_{0}^{I} .
$$

Let $\beta=w_{2}\left(\alpha_{i}\right)$. Then $\beta \in \mathbb{N}_{0} \alpha_{i}+\mathbb{N}_{0} \alpha_{j}$, since $\ell\left(w_{2} \sigma_{i}\right)>\ell\left(w_{2}\right)$. Moreover, $\beta$ is not simple. Indeed, $\alpha=w\left(\alpha_{i}\right)=w_{1}(\beta)$, so $\beta$ is not simple, since $\ell\left(w_{1}\right)<\ell(w)$ and $\ell(w)$ was chosen of minimal length. By Proposition 2.7 we conclude that $\beta$ is the sum of two positive roots $\beta_{1}, \beta_{2} \in \mathbb{N}_{0} \alpha_{i}+\mathbb{N}_{0} \alpha_{j}$. It remains to check that $w_{1}\left(\beta_{1}\right)$, $w_{1}\left(\beta_{2}\right)$ are positive. But this follows from (2.3). 


\section{OBstructions FOR WEYL GROUPOIDS OF RANK THREE}

In this section we analyze the structure of finite Weyl groupoids of rank three. Let $\mathcal{C}$ be a Cartan scheme of rank three, and assume that $\mathcal{R}^{\text {re }}(\mathcal{C})$ is a finite irreducible root system of type $\mathcal{C}$. In this case a hyperplane in $\mathbb{Z}^{I}$ is the same as a cofree subgroup of rank two, which will be called a plane in the sequel. For simplicity we will take $I=\{1,2,3\}$, and we write $R_{+}^{a}$ for the set of positive real roots at $a \in A$.

Recall the definition of the functions $\operatorname{Vol}_{k}$, where $k \in\{1,2,3\}$, from the previous section. As noted, for three elements $\alpha, \beta, \gamma \in \mathbb{Z}^{3}$ we have $\operatorname{Vol}_{3}(\alpha, \beta, \gamma)=1$ if and only if $\{\alpha, \beta, \gamma\}$ is a basis of $\mathbb{Z}^{3}$. Also, we will heavily use the notion of a base; see Definition 2.3 .

Lemma 3.1. Let $a \in A$ and $\alpha, \beta \in R^{a}$. Assume that $\alpha \neq \pm \beta$ and that $\{\alpha, \beta\}$ is not a base for $V^{a}(\alpha, \beta)$ at a. Then there exist $k, l \in \mathbb{N}$ and $\delta \in R^{a}$ such that $\beta-k \alpha=l \delta$ and $\{\alpha, \delta\}$ is a base for $V^{a}(\alpha, \beta)$ at $a$.

Proof. By Theorem 2.4 there exists an object $b$ and $w \in \operatorname{Hom}(a, b)$ such that $w(\alpha)$ is simple and $w(\beta)=k w(\alpha)+l w(\delta)$ for some simple root $w(\delta)$ and $k, l \in \mathbb{Z}_{\geq 0}$. Thus $\beta=k \alpha+l \delta$. The relation $\beta \neq \delta$ follows from the assumption that $\{\alpha, \beta\}$ is not a base for $V^{a}(\alpha, \beta)$ at $a$.

Lemma 3.2. Let $a \in A$ and $\alpha, \beta \in R^{a}$ such that $\alpha \neq \pm \beta$. Then $\{\alpha, \beta\}$ is a base for $V^{a}(\alpha, \beta)$ if and only if $\operatorname{Vol}_{2}(\alpha, \beta)=1$ and $\alpha-\beta \notin R^{a}$.

Proof. Assume first that $\{\alpha, \beta\}$ is a base for $V^{a}(\alpha, \beta)$ at $a$. By Corollary 2.5 we may assume that $\alpha$ and $\beta$ are simple roots. Therefore $\operatorname{Vol}_{2}(\alpha, \beta)=1$ and $\alpha-\beta \notin R^{a}$.

Conversely, assume that $\operatorname{Vol}_{2}(\alpha, \beta)=1, \alpha-\beta \notin R^{a}$, and that $\{\alpha, \beta\}$ is not a base for $V^{a}(\alpha, \beta)$ at $a$. Let $k, l, \delta$ be as in Lemma 3.1, Then

$$
1=\operatorname{Vol}_{2}(\alpha, \beta)=\operatorname{Vol}_{2}(\alpha, \beta-k \alpha)=l \operatorname{Vol}_{2}(\alpha, \delta) .
$$

Hence $l=1$, and $\{\alpha, \delta\}=\{\alpha, \beta-k \alpha\}$ is a base for $V^{a}(\alpha, \beta)$ at $a$. Then $\beta-\alpha \in R^{a}$ by Corollary 2.8 and since $k>0$. This gives the desired contradiction to the assumption $\alpha-\beta \notin R^{a}$.

Recall that a semigroup ordering $<$ on a commutative semigroup $(S,+)$ is a total ordering such that for all $s, t, u \in S$ with $s<t$ the relations $s+u<t+u$ hold. For example, the lexicographic ordering on $\mathbb{Z}^{I}$ induced by any total ordering on $I$ is a semigroup ordering.

Lemma 3.3. Let $a \in A$, and let $V \subset \mathbb{Z}^{I}$ be a plane containing at least two positive roots of $R^{a}$. Let $<$ be a semigroup ordering on $\mathbb{Z}^{I}$ such that $0<\gamma$ for all $\gamma \in R_{+}^{a}$, and let $\alpha, \beta$ denote the two smallest elements in $V \cap R_{+}^{a}$ with respect to $<$. Then $\{\alpha, \beta\}$ is a base for $V$ at $a$.

Proof. Let $\alpha$ be the smallest element of $V \cap R_{+}^{a}$ with respect to $<$, and let $\beta$ be the smallest element of $V \cap\left(R_{+}^{a} \backslash\{\alpha\}\right)$. Then $V=V^{a}(\alpha, \beta)$ by (R2). By Lemma 3.1 there exists $\delta \in V \cap R^{a}$ such that $\{\alpha, \delta\}$ is a base for $V$ at $a$. First suppose that $\delta<0$. Let $m \in \mathbb{N}_{0}$ be the smallest integer with $\delta+(m+1) \alpha \notin R^{a}$. Then $\delta+n \alpha<0$ for all $n \in \mathbb{N}_{0}$ with $n \leq m$. Indeed, this holds for $n=0$ by assumption. By induction on $n$ we obtain from $\delta+n \alpha<0$ and the choice of $\alpha$ that $\delta+n \alpha<-\alpha$, since $\delta$ and $\alpha$ are not collinear. Hence $\delta+(n+1) \alpha<0$. We conclude that $-(\delta+m \alpha)>0$. Moreover, $\{\alpha,-(\delta+m \alpha)\}$ is a base for $V$ at $a$ by Lemma 3.2 and the choice of $m$. Therefore, by replacing $\{\alpha, \delta\}$ by $\{\alpha,-(\delta+m \alpha)\}$, we may assume that $\delta>0$. Since 
$\beta>0$, we conclude that $\beta=k \alpha+l \delta$ for some $k, l \in \mathbb{N}_{0}$. Since $\beta$ is not a multiple of $\alpha$, this implies that $\beta=\delta$ or $\beta>\delta$. Then the choice of $\beta$ and the positivity of $\delta$ yield that $\delta=\beta$; that is, $\{\alpha, \beta\}$ is a base for $V$ at $a$.

Lemma 3.4. Let $k \in \mathbb{N}_{\geq 2}, a \in A, \alpha \in R_{+}^{a}$, and $\beta \in \mathbb{Z}^{I}$ such that $\alpha$ and $\beta$ are not collinear and $\alpha+k \beta \in R^{a}$. Assume that $\operatorname{Vol}_{2}(\alpha, \beta)=1$ and that $(-\mathbb{N} \alpha+\mathbb{Z} \beta) \cap \mathbb{N}_{0}^{I}=$ $\emptyset$. Then $\beta \in R^{a}$ and $\alpha+l \beta \in R^{a}$ for all $l \in\{1,2, \ldots, k\}$.

Proof. We prove the claim indirectly. Assume that $\beta \notin R^{a}$. By Lemma 3.3 there exists a base $\left\{\gamma_{1}, \gamma_{2}\right\}$ for $V^{a}(\alpha, \beta)$ at a such that $\gamma_{1}, \gamma_{2} \in R_{+}^{a}$. The assumptions of the lemma imply that there exist $m_{1}, l_{1} \in \mathbb{N}_{0}$ and $m_{2}, l_{2} \in \mathbb{Z}$ such that $\gamma_{1}=$ $m_{1} \alpha+m_{2} \beta, \gamma_{2}=l_{1} \alpha+l_{2} \beta$. Since $\beta \notin R^{a}$, we obtain that $m_{1} \geq 1$ and $m_{2} \geq 1$. Therefore relations $\alpha, \alpha+k \beta \in R_{+}^{a}$ imply that $\{\alpha, \alpha+k \beta\}=\left\{\gamma_{1}, \gamma_{2}\right\}$. The latter is a contradiction to $\operatorname{Vol}_{2}\left(\gamma_{1}, \gamma_{2}\right)=1$ and $\operatorname{Vol}_{2}(\alpha, \alpha+k \beta)=k>1$. Thus $\beta \in R^{a}$. By Lemma 3.1 we obtain that $\{\beta, \alpha-m \beta\}$ is a base for $V^{a}(\alpha, \beta)$ at a for some $m \in \mathbb{N}_{0}$. Then Corollary 2.8 and the assumption that $\alpha+k \beta \in R^{a}$ imply the last claim of the lemma.

We say that a subset $S$ of $\mathbb{Z}^{3}$ is convex if any rational convex linear combination of elements of $S$ is either in $S$ or not in $\mathbb{Z}^{3}$. We start with a simple example.

Lemma 3.5. Let $a \in A$. Assume that $c_{12}^{a}=0$.

(1) Let $k_{1}, k_{2} \in \mathbb{Z}$. Then $\alpha_{3}+k_{1} \alpha_{1}+k_{2} \alpha_{2} \in R^{a}$ if and only if $0 \leq k_{1} \leq-c_{13}^{a}$ and $0 \leq k_{2} \leq-c_{23}^{a}$.

(2) Let $\gamma \in\left(\alpha_{3}+\mathbb{Z} \alpha_{1}+\mathbb{Z} \alpha_{2}\right) \cap R^{a}$. Then $\gamma-\alpha_{1} \in R^{a}$ or $\gamma+\alpha_{1} \in R^{a}$. Similarly $\gamma-\alpha_{2} \in R^{a}$ or $\gamma+\alpha_{2} \in R^{a}$.

Proof. (1) The assumption $c_{12}^{a}=0$ implies $c_{23}^{\rho_{1}(a)}=c_{23}^{a}$; see [CH09c, Lemma 4.5]. Applying $\sigma_{1}^{\rho_{1}(a)}, \sigma_{2}^{\rho_{2}(a)}$, and $\sigma_{1} \sigma_{2}^{\rho_{2} \rho_{1}(a)}$ to $\alpha_{3}$ we conclude that $\alpha_{3}-c_{13}^{a} \alpha_{1}, \alpha_{3}-c_{23}^{a} \alpha_{2}$, $\alpha_{3}-c_{13}^{a} \alpha_{1}-c_{23}^{a} \alpha_{2} \in R_{+}^{a}$. Thus Lemma 3.4 implies that $\alpha_{3}+m_{1} \alpha_{1}+m_{2} \alpha_{2} \in R^{a}$ for all $m_{1}, m_{2} \in \mathbb{Z}$ with $0 \leq m_{1} \leq-c_{13}^{a}$ and $0 \leq m_{2} \leq-c_{23}^{a}$. Further, (R1) gives that $\alpha_{3}+k_{1} \alpha_{1}+k_{2} \alpha_{2} \notin R^{a}$ if $k_{1}<0$ or $k_{2}<0$. Applying again the simple reflections $\sigma_{1}$ and $\sigma_{2}$, a similar argument proves the remaining part of the claim. Observe that the proof does not use the fact that $\mathcal{R}^{\text {re }}(\mathcal{C})$ is irreducible.

(2) Since $c_{12}^{a}=0$, the irreducibility of $\mathcal{R}^{\text {re }}(\mathcal{C})$ yields that $c_{13}^{a}, c_{23}^{a}<0$ by $\mathrm{CH} 09 \mathrm{c}$, Def. 4.5, Prop. 4.6]. Hence the claim follows from (1).

Proposition 3.6. Let $a \in A$ and let $\gamma_{1}, \gamma_{2}, \gamma_{3} \in R^{a}$. Assume that $\operatorname{Vol}_{3}\left(\gamma_{1}, \gamma_{2}, \gamma_{3}\right)=$ 1 and that $\gamma_{1}-\gamma_{2}, \gamma_{1}-\gamma_{3} \notin R^{a}$. Then $\gamma_{1}+\gamma_{2} \in R^{a}$ or $\gamma_{1}+\gamma_{3} \in R^{a}$.

Proof. Since $\gamma_{1}-\gamma_{2} \notin R^{a}$ and $\operatorname{Vol}_{3}\left(\gamma_{1}, \gamma_{2}, \gamma_{3}\right)=1$, Theorem 2.4 and Lemma 3.2 imply that there exists $b \in A, w \in \operatorname{Hom}(a, b)$ and $i_{1}, i_{2}, i_{3} \in I$ such that $w\left(\gamma_{1}\right)=\alpha_{i_{1}}$, $w\left(\gamma_{2}\right)=\alpha_{i_{2}}$, and $w\left(\gamma_{3}\right)=\alpha_{i_{3}}+k_{1} \alpha_{i_{1}}+k_{2} \alpha_{i_{2}}$ for some $k_{1}, k_{2} \in \mathbb{N}_{0}$. Assume that $\gamma_{1}+\gamma_{2} \notin R^{a}$. Then $c_{i_{1} i_{2}}^{b}=0$. Since $\gamma_{3}-\gamma_{1} \notin R^{a}$, Lemma 3.5(2) with $\gamma=w\left(\gamma_{3}\right)$ gives that $\gamma_{3}+\gamma_{1} \in R^{a}$. This proves the claim.

Lemma 3.7. Assume that $R^{a} \cap\left(\mathbb{N}_{0} \alpha_{1}+\mathbb{N}_{0} \alpha_{2}\right)$ contains at most 4 positive roots.

(1) The set $S_{3}:=\left(\alpha_{3}+\mathbb{Z} \alpha_{1}+\mathbb{Z} \alpha_{2}\right) \cap R^{a}$ is convex.

(2) Let $\gamma \in S_{3}$. Then $\gamma=\alpha_{3}$ or $\gamma-\alpha_{1} \in R^{a}$ or $\gamma-\alpha_{2} \in R^{a}$.

Proof. Consider the roots of the form $w^{-1}\left(\alpha_{3}\right) \in R^{a}$, where $w \in \operatorname{Hom}(a, \mathcal{W}(\mathcal{C}))$ is a product of reflections $\sigma_{1}^{b}, \sigma_{2}^{b}$ with $b \in A$. All of these roots belong to $S_{3}$. Using 
Lemma 3.4 the claims of the lemma can be checked case by case, similarly to the proof of Lemma 3.5.

Remark 3.8. The lemma can be proven by elementary calculations, since all nonsimple positive roots in $\left(\mathbb{Z} \alpha_{1}+\mathbb{Z} \alpha_{2}\right) \cap R^{a}$ are of the form say $\alpha_{1}+k \alpha_{2}, k \in \mathbb{N}$ when $\#\left(R^{a} \cap\left(\mathbb{N}_{0} \alpha_{1}+\mathbb{N}_{0} \alpha_{2}\right)\right) \leq 4$. We will see in Theorem 4.1 that the classification of connected Cartan schemes of rank three admitting a finite irreducible root system has a finite set of solutions. Thus it is possible to check the claim of the lemma for any such Cartan scheme. Using computer calculations one obtains that the lemma holds without any restriction on the (finite) cardinality of $R^{a} \cap\left(\mathbb{N}_{0} \alpha_{1}+\mathbb{N}_{0} \alpha_{2}\right)$.

Lemma 3.9. Let $\alpha, \beta, \gamma \in R^{a}$ such that $\operatorname{Vol}_{3}(\alpha, \beta, \gamma)=1$. Assume that $\alpha-\beta$, $\beta-\gamma, \alpha-\gamma \notin R^{a}$ and that $\{\alpha, \beta, \gamma\}$ is not a base for $\mathbb{Z}^{I}$ at a. Then the following hold.

(1) There exist $w \in \operatorname{Hom}(a, \mathcal{W}(\mathcal{C}))$ and $n_{1}, n_{2} \in \mathbb{N}$ such that $w(\alpha), w(\beta)$, and $w\left(\gamma-n_{1} \alpha-n_{2} \beta\right)$ are simple roots.

(2) None of the vectors $\alpha-k \beta, \alpha-k \gamma, \beta-k \alpha, \beta-k \gamma, \gamma-k \alpha, \gamma-k \beta$, where $k \in \mathbb{N}$, is contained in $R^{a}$.

(3) $\alpha+\beta, \alpha+\gamma, \beta+\gamma \in R^{a}$.

(4) One of the sets $\{\alpha+2 \beta, \beta+2 \gamma, \gamma+2 \alpha\}$ and $\{2 \alpha+\beta, 2 \beta+\gamma, 2 \gamma+\alpha\}$ is contained in $R^{a}$, the other one has trivial intersection with $R^{a}$.

(5) None of the vectors $\gamma-\alpha-k \beta, \gamma-k \alpha-\beta, \beta-\gamma-k \alpha, \beta-k \gamma-\alpha, \alpha-\beta-k \gamma$, $\alpha-k \beta-\gamma$, where $k \in \mathbb{N}_{0}$, is contained in $R^{a}$.

(6) Assume that $\alpha+2 \beta \in R^{a}$. Let $k \in \mathbb{N}$ such that $\alpha+k \beta \in R^{a}, \alpha+(k+1) \beta \notin R^{a}$. Let $\alpha^{\prime}=\alpha+k \beta, \beta^{\prime}=-\beta, \gamma^{\prime}=\gamma+\beta$. Then $\operatorname{Vol}_{3}\left(\alpha^{\prime}, \beta^{\prime}, \gamma^{\prime}\right)=1,\left\{\alpha^{\prime}, \beta^{\prime}, \gamma^{\prime}\right\}$ is not a base for $\mathbb{Z}^{I}$ at $a$, and none of $\alpha^{\prime}-\beta^{\prime}, \alpha^{\prime}-\gamma^{\prime}, \beta^{\prime}-\gamma^{\prime}$ is contained in $R^{a}$.

(7) None of the vectors $\alpha+3 \beta, \beta+3 \gamma, \gamma+3 \alpha, 3 \alpha+\beta, 3 \beta+\gamma, 3 \gamma+\alpha$ is contained in $R^{a}$. In particular, $k=2$ holds in (6).

Proof. (1) By Theorem 2.4 there exist $m_{1}, m_{2}, n_{1}, n_{2}, n_{3} \in \mathbb{N}_{0}, i_{1}, i_{2}, i_{3} \in I$, and $w \in \operatorname{Hom}(a, \mathcal{W}(\mathcal{C}))$ such that $w(\alpha)=\alpha_{i_{1}}, w(\beta)=m_{1} \alpha_{i_{1}}+m_{2} \alpha_{i_{2}}$, and $w(\gamma)=$ $n_{1} \alpha_{i_{1}}+n_{2} \alpha_{i_{2}}+n_{3} \alpha_{i_{3}}$. Since $\operatorname{det} w \in\{ \pm 1\}$ and $\operatorname{Vol}_{3}(\alpha, \beta, \gamma)=1$, this implies that $m_{2}=n_{3}=1$. Further, $\beta-\alpha \notin R^{a}$, and hence $w(\beta)=\alpha_{i_{2}}$ by Corollary 2.9. Since $\{\alpha, \beta, \gamma\}$ is not a base for $\mathbb{Z}^{I}$ at $a$, we conclude that $w(\gamma) \neq \alpha_{i_{3}}$. Then Corollary 2.9 and the assumptions $\gamma-\alpha, \gamma-\beta \notin R^{a}$ imply that $w(\gamma) \notin \alpha_{i_{3}}+\mathbb{N}_{0} \alpha_{i_{1}}$ and $w(\gamma) \notin \alpha_{i_{3}}+\mathbb{N}_{0} \alpha_{i_{2}}$. Thus the claim is proven.

(2) By (1), $\{\alpha, \beta\}$ is a base for $V^{a}(\alpha, \beta)$ at $a$. Thus $\alpha-k \beta \notin R^{a}$ for all $k \in \mathbb{N}$. The remaining claims follow by symmetry.

(3) Suppose that $\alpha+\beta \notin R^{a}$. By (1) there exist $b \in A, w \in \operatorname{Hom}(a, b), i_{1}, i_{2}, i_{3} \in$ $I$ and $n_{1}, n_{2} \in \mathbb{N}$ such that $w(\alpha)=\alpha_{i_{1}}, w(\beta)=\alpha_{i_{2}}$, and $w(\gamma)=\alpha_{i_{3}}+n_{1} \alpha_{i_{1}}+$ $n_{2} \alpha_{i_{2}} \in R_{+}^{b}$. By Theorem 2.10 there exist $n_{1}^{\prime}, n_{2}^{\prime} \in \mathbb{N}_{0}$ such that $n_{1}^{\prime} \leq n_{1}, n_{2}^{\prime} \leq n_{2}$, $n_{1}^{\prime}+n_{2}^{\prime}<n_{1}+n_{2}$, and

$$
\alpha_{i_{3}}+n_{1}^{\prime} \alpha_{i_{1}}+n_{2}^{\prime} \alpha_{i_{2}} \in R_{+}^{b}, \quad\left(n_{1}-n_{1}^{\prime}\right) \alpha_{i_{1}}+\left(n_{2}-n_{2}^{\prime}\right) \alpha_{i_{2}} \in R_{+}^{b} .
$$

Since $\alpha+\beta \notin R^{a}$, Proposition 2.6 yields that $R_{+}^{b} \cap \operatorname{span}_{\mathbb{Z}}\left\{\alpha_{i_{1}}, \alpha_{i_{2}}\right\}=\left\{\alpha_{i_{1}}, \alpha_{i_{2}}\right\}$. Thus $\gamma-\alpha \in R^{a}$ or $\gamma-\beta \in R^{a}$. This is a contradiction to the assumption of the lemma. Hence $\alpha+\beta \in R^{a}$. By symmetry we obtain that $\alpha+\gamma, \beta+\gamma \in R^{a}$.

(4) Suppose that $\alpha+2 \beta, 2 \alpha+\beta \notin R^{a}$. By (1) the set $\{\alpha, \beta\}$ is a base for $V^{a}(\alpha, \beta)$ at $a$, and $\alpha+\beta \in R^{a}$ by (3). Then Proposition 2.6 implies that $R^{a} \cap \operatorname{span}_{\mathbb{Z}}\{\alpha, \beta\}=$ $\{ \pm \alpha, \pm \beta, \pm(\alpha+\beta)\}$. Thus (1) and Lemma $3.7(2)$ give that $\gamma-\alpha \in R^{a}$ or $\gamma-\beta \in R^{a}$, 
a contradiction to the initial assumption of the lemma. Hence by symmetry each of the sets $\{\alpha+2 \beta, 2 \alpha+\beta\},\{\alpha+2 \gamma, 2 \alpha+\gamma\},\{\beta+2 \gamma, 2 \beta+\gamma\}$ contains at least one element of $R^{a}$.

Assume now that $\gamma+2 \alpha, \gamma+2 \beta \in R^{a}$. By changing the object via (1) we may assume that $\alpha, \beta$, and $\gamma-n_{1} \alpha-n_{2} \beta$ are simple roots for some $n_{1}, n_{2} \in \mathbb{N}$. Then Lemma 3.4 applies to $\gamma+2 \alpha \in R_{+}^{a}$ and $\beta-\alpha$ and tells us that $\beta-\alpha \in R^{a}$. This gives a contradiction.

By the previous two paragraphs we conclude that if $\gamma+2 \alpha \in R^{a}$, then $\gamma+2 \beta \notin$ $R^{a}$, and hence $\beta+2 \gamma \in R^{a}$. Similarly, we also obtain that $\alpha+2 \beta \in R^{a}$. By symmetry this implies (4).

(5) By symmetry it suffices to prove that $\gamma-(\alpha+k \beta) \notin R^{a}$ for all $k \in \mathbb{N}_{0}$. For $k=0$ the claim holds by assumption.

First we prove that $\gamma-(\alpha+2 \beta) \notin R^{a}$. By (3) we know that $\gamma+\alpha, \alpha+\beta \in R^{a}$, and $\gamma-\beta \notin R^{a}$ by assumption. Since $\operatorname{Vol}_{2}(\gamma+\alpha, \alpha+\beta)=1$, Lemma 3.2 gives that $\{\gamma+\alpha, \alpha+\beta\}$ is a base for $V^{a}(\gamma+\alpha, \alpha+\beta)$ at $a$. Since $\gamma-(\alpha+2 \beta)=(\gamma+\alpha)-2(\alpha+\beta)$, we conclude that $\gamma-(\alpha+2 \beta) \notin R^{a}$.

Now let $k \in \mathbb{N}$. Assume that $\gamma-(\alpha+k \beta) \in R^{a}$ and that $k$ is minimal with this property. Let $\alpha^{\prime}=-\alpha, \beta^{\prime}=-\beta, \gamma^{\prime}=\gamma-(\alpha+k \beta)$. Then $\alpha^{\prime}, \beta^{\prime}, \gamma^{\prime} \in R^{a}$ with $\operatorname{Vol}_{3}\left(\alpha^{\prime}, \beta^{\prime}, \gamma^{\prime}\right)=1$. Moreover, $\alpha^{\prime}-\beta^{\prime} \notin R^{a}$ by assumption, $\alpha^{\prime}-\gamma^{\prime}=-(\gamma-k \beta) \notin R^{a}$ by $(2)$, and $\beta^{\prime}-\gamma^{\prime}=-(\gamma-\alpha-(k-1) \beta) \notin R^{a}$ by the minimality of $k$. Further, $\left\{\alpha^{\prime}, \beta^{\prime}, \gamma^{\prime}\right\}$ is not a base for $R^{a}$, since $\gamma=\gamma^{\prime}-\alpha^{\prime}-k \beta^{\prime}$. Hence Claim (3) holds for $\alpha^{\prime}, \beta^{\prime}, \gamma^{\prime}$. In particular,

$$
\gamma^{\prime}+\beta^{\prime}=\gamma-(\alpha+(k+1) \beta) \in R^{a}
$$

This and the previous paragraph imply that $k \geq 3$.

We distinguish two cases depending on the parity of $k$. First assume that $k$ is even. Let $\alpha^{\prime}=\gamma+\alpha$ and $\beta^{\prime}=-(\alpha+k / 2 \beta)$. Then $\operatorname{Vol}_{2}\left(\alpha^{\prime}, \beta^{\prime}\right)=1$ and $\alpha^{\prime}+2 \beta^{\prime}=$ $\gamma-(\alpha+k \beta) \in R^{a}$. Lemma 3.4 applied to $\alpha^{\prime}, \beta^{\prime}$ gives that $\gamma-k / 2 \beta=\alpha^{\prime}+\beta^{\prime} \in R^{a}$, which contradicts $(2)$.

Finally, the case of odd $k$ can be excluded similarly by the consideration of $V^{a}(\gamma+\alpha, \gamma-(\alpha+(k+1) \beta))$.

(6) We get $\operatorname{Vol}_{3}\left(\alpha^{\prime}, \beta^{\prime}, \gamma^{\prime}\right)=1$ since $\operatorname{Vol}_{3}(\alpha, \beta, \gamma)=1$ and $\operatorname{Vol}_{3}$ is invariant under the right action of $\operatorname{GL}\left(\mathbb{Z}^{3}\right)$. Further, $\beta^{\prime}-\gamma^{\prime}=-(2 \beta+\gamma) \notin R^{a}$ by (4), and $\alpha^{\prime}-\gamma^{\prime} \notin R^{a}$ by (5). Finally, $\left(\alpha^{\prime}, \beta^{\prime}, \gamma^{\prime}\right)$ is not a base for $\mathbb{Z}^{I}$ at $a$, since $R^{a} \ni \gamma-n_{1} \alpha-n_{2} \beta=\gamma^{\prime}-n_{1} \alpha^{\prime}+\left(1+n_{2}-k n_{1}\right) \beta^{\prime}$, where $n_{1}, n_{2} \in \mathbb{N}$ are as in (1).

(7) We prove that $\gamma+3 \alpha \notin R^{a}$. The rest follows by symmetry. If $2 \alpha+\beta \in R^{a}$, then $\gamma+2 \alpha \notin R^{a}$ by (4), and hence $\gamma+3 \alpha \notin R^{a}$. Otherwise $\alpha+2 \beta, \gamma+2 \alpha \in R^{a}$ by (4). Let $k, \alpha^{\prime}, \beta^{\prime}, \gamma^{\prime}$ be as in (6). Then (6) and (3) give that $R^{a} \ni \gamma^{\prime}+\alpha^{\prime}=$ $\gamma+\alpha+(k+1) \beta$. Since $\gamma+\alpha \in R^{a}$, Lemma 3.4 implies that $\gamma+\alpha+2 \beta \in R^{a}$. Let $w$ be as in (1). If $\gamma+3 \alpha \in R^{a}$, then Lemma 3.4 for the vectors $w(\gamma+\alpha+2 \beta)$ and $w(\alpha-\beta)$ implies that $w(\alpha-\beta) \in R^{a}$, a contradiction. Thus $\gamma+3 \alpha \notin R^{a}$.

Recall that $\mathcal{C}$ is a Cartan scheme of rank three and $\mathcal{R}^{\text {re }}(\mathcal{C})$ is a finite irreducible root system of type $\mathcal{C}$.

Theorem 3.10. Let $a \in A$ and $\alpha, \beta, \gamma \in R^{a}$. If $\operatorname{Vol}_{3}(\alpha, \beta, \gamma)=1$ and none of $\alpha-\beta, \alpha-\gamma, \beta-\gamma$ are contained in $R^{a}$, then $\{\alpha, \beta, \gamma\}$ is a base for $\mathbb{Z}^{I}$ at a.

Proof. Assume to the contrary that $\{\alpha, \beta, \gamma\}$ is not a base for $\mathbb{Z}^{I}$ at $a$. Exchanging $\alpha$ and $\beta$ if necessary, by Lemma $3.9(4)$ we may assume that $\alpha+2 \beta \in R^{a}$. 
By Lemma $3.9(6),(7)$ the triple $(\alpha+2 \beta,-\beta, \gamma+\beta)$ satisfies the assumptions of Lemma 3.9 and $(\alpha+2 \beta)+2(-\beta)=\alpha \in R^{a}$. Hence $2 \alpha+3 \beta=2(\alpha+2 \beta)+(-\beta) \notin R^{a}$ by Lemma 3.9. (4). Thus $V^{a}(\alpha, \beta) \cap R^{a}=\{ \pm \alpha, \pm(\alpha+\beta), \pm(\alpha+2 \beta), \pm \beta\}$ by Proposition 2.6. and hence, using Lemma 3.9(1), we obtain from Lemma 3.7(2) that $\gamma-\alpha \in R^{a}$ or $\gamma-\beta \in R^{a}$. This is a contradiction to our initial assumption, and hence $\{\alpha, \beta, \gamma\}$ is a base for $\mathbb{Z}^{I}$ at $a$.

Corollary 3.11. Let $a \in A$ and $\gamma_{1}, \gamma_{2}, \alpha \in R^{a}$. Assume that $\left\{\gamma_{1}, \gamma_{2}\right\}$ is a base for $V^{a}\left(\gamma_{1}, \gamma_{2}\right)$ at a and that $\operatorname{Vol}_{3}\left(\gamma_{1}, \gamma_{2}, \alpha\right)=1$. Then either $\left\{\gamma_{1}, \gamma_{2}, \alpha\right\}$ is a base for $\mathbb{Z}^{I}$ at $a$, or one of $\alpha-\gamma_{1}, \alpha-\gamma_{2}$ is contained in $R^{a}$.

For the proof of Theorem 4.1 we need a bound for the entries of the Cartan matrices of $\mathcal{C}$. To get this bound we use the following.

Lemma 3.12. Let $a \in A$.

(1) At most one of $c_{12}^{a}, c_{13}^{a}, c_{23}^{a}$ is zero.

(2) $\alpha_{1}+\alpha_{2}+\alpha_{3} \in R^{a}$.

(3) Let $k \in \mathbb{Z}$. Then $k \alpha_{1}+\alpha_{2}+\alpha_{3} \in R^{a}$ if and only if $k_{1} \leq k \leq k_{2}$, where

$$
k_{1}=\left\{\begin{array}{ll}
0 & \text { if } c_{23}^{a}<0, \\
1 & \text { if } c_{23}^{a}=0,
\end{array} \quad k_{2}= \begin{cases}-c_{12}^{a}-c_{13}^{a} & \text { if } c_{23}^{\rho_{1}(a)}<0, \\
-c_{12}^{a}-c_{13}^{a}-1 & \text { if } c_{23}^{\rho_{1}(a)}=0 .\end{cases}\right.
$$

(4) We have $2 \alpha_{1}+\alpha_{2}+\alpha_{3} \in R^{a}$ if and only if either $c_{12}^{a}+c_{13}^{a} \leq-3$ or $c_{12}^{a}+c_{13}^{a}=$ $-2, c_{23}^{\rho_{1}(a)}<0$.

(5) Assume that

$$
\#\left(R_{+}^{a} \cap\left(\mathbb{Z} \alpha_{1}+\mathbb{Z} \alpha_{2}\right)\right) \geq 5 .
$$

Then there exist $k \in \mathbb{N}_{0}$ such that $k \alpha_{1}+2 \alpha_{2}+\alpha_{3} \in R^{a}$. Let $k_{0}$ be the smallest among all such $k$. Then $k_{0}$ is given by the following:

$$
\left\{\begin{array}{l}
0 \quad \text { if } c_{23}^{a} \leq-2, \\
1 \quad \text { if }-1 \leq c_{23}^{a} \leq 0, c_{21}^{a}+c_{23}^{a} \leq-2, c_{13}^{\rho_{2}(a)}<0, \\
1 \quad \text { if }-1 \leq c_{23}^{a} \leq 0, c_{21}^{a}+c_{23}^{a} \leq-3, c_{13}^{\rho_{2}(a)}=0 \\
2 \quad \text { if } c_{21}^{a}=c_{23}^{a}=-1, c_{13}^{\rho_{2}(a)}=0 \\
2 \quad \text { if } c_{21}^{a}=-1, c_{23}^{a}=0, c_{13}^{\rho_{2}(a)} \leq-2, \\
3 \quad \text { if } c_{21}^{a}=-1, c_{23}^{a}=0, c_{13}^{\rho_{2}(a)}=-1, c_{12}^{\rho_{2}(a)} \leq-3, \\
3 \quad \text { if } c_{21}^{a}=-1, c_{23}^{a}=0, c_{13}^{\rho_{2}(a)}=-1, c_{12}^{\rho_{2}(a)}=-2, c_{23}^{\rho_{1} \rho_{2}(a)}<0, \\
4 \quad \text { otherwise. }
\end{array}\right.
$$

Further, if $c_{13}^{a}=0$, then $k_{0} \leq 2$.

Proof. We may assume that $\mathcal{C}$ is connected. Then, since $\mathcal{R}^{\mathrm{re}}(\mathcal{C})$ is irreducible, Claim (1) holds by [CH09c, Def. 4.5, Prop. 4.6].

(2) The claim is invariant under permutations of $I$. Thus by (1) we may assume that $c_{23}^{a} \neq 0$. Hence $\alpha_{2}+\alpha_{3} \in R^{a}$. Assume first that $c_{13}^{a}=0$. Then $c_{13}^{\rho_{1}(a)}=0$ by $(\mathrm{C} 2), c_{23}^{\rho_{1}(a)} \neq 0$ by (1), and $\alpha_{2}+\alpha_{3} \in R_{+}^{\rho_{1}(a)}$. Hence $\sigma_{1}^{\rho_{1}(a)}\left(\alpha_{2}+\alpha_{3}\right)=$ $-c_{12}^{a} \alpha_{1}+\alpha_{2}+\alpha_{3} \in R^{a}$. Therefore (2) holds by Lemma 3.4 for $\alpha=\alpha_{2}+\alpha_{3}$ and $\beta=\alpha_{1}$.

Assume now that $c_{13}^{a} \neq 0$. By symmetry and the previous paragraph we may also assume that $c_{12}^{a}, c_{23}^{a} \neq 0$. Let $b=\rho_{1}(a)$. If $c_{23}^{b}=0$, then $\alpha_{1}+\alpha_{2}+\alpha_{3} \in R^{b}$ by 
the previous paragraph. Then

$$
R^{a} \ni \sigma_{1}^{b}\left(\alpha_{1}+\alpha_{2}+\alpha_{3}\right)=\left(-c_{12}^{a}-c_{13}^{a}-1\right) \alpha_{1}+\alpha_{2}+\alpha_{3},
$$

and the coefficient of $\alpha_{1}$ is positive. Further, $\alpha_{2}+\alpha_{3} \in R^{a}$, and hence (2) holds in this case by Lemma 3.4. Finally, if $c_{23}^{b} \neq 0$, then $\alpha_{2}+\alpha_{3} \in R_{+}^{b}$, and hence $\left(-c_{12}^{a}-c_{13}^{a}\right) \alpha_{1}+\alpha_{2}+\alpha_{3} \in R^{a}$. Since $-c_{12}^{a}-c_{13}^{a}>0$, (2) follows again from Lemma 3.4

(3) If $c_{23}^{a}<0$, then $\alpha_{2}+\alpha_{3} \in R^{a}$ and $-k \alpha_{1}+\alpha_{2}+\alpha_{3} \notin R^{a}$ for all $k \in \mathbb{N}$. If $c_{23}^{a}=0$, then $\alpha_{1}+\alpha_{2}+\alpha_{3} \in R^{a}$ by (2), and $-k \alpha_{1}+\alpha_{2}+\alpha_{3} \notin R^{a}$ for all $k \in \mathbb{N}_{0}$. Applying the same argument to $R^{\rho_{1}(a)}$ and using the reflection $\sigma_{1}^{\rho_{1}(a)}$ and Lemma 3.4 gives the claim.

(4) This follows immediately from (3).

(5) The first case follows from Corollary 2.9 and the second and third cases are obtained from (4) by interchanging the elements 1 and 2 of $I$. We also obtain that if $k_{0}$ exists, then $k_{0} \geq 2$ in all other cases. By (3.1) and Proposition 2.6 we conclude that $\alpha_{1}+\alpha_{2} \in R^{a}$. Then $c_{21}^{a}<0$ by Corollary [2.9, and hence we are left with calculating $k_{0}$ if $-1 \leq c_{23}^{a} \leq 0, c_{21}^{a}+c_{23}^{a}=-2, c_{13}^{\rho_{2}(a)}=0$, or $c_{21}^{a}=-1, c_{23}^{a}=0$. By (1), if $c_{13}^{\rho_{2}(a)}=0$, then $c_{23}^{\rho_{2}(a)} \neq 0$, and hence $c_{23}^{a}<0$ by (C2). Thus we have to consider the elements $k \alpha_{1}+2 \alpha_{2}+\alpha_{3}$, where $k \geq 2$, under the assumption that

$$
c_{21}^{a}=c_{23}^{a}=-1, c_{13}^{\rho_{2}(a)}=0 \quad \text { or } \quad c_{21}^{a}=-1, c_{23}^{a}=0 .
$$

Since $c_{21}^{a}=-1$, Condition (3.1) gives that

$$
c_{12}^{\rho_{2}(a)} \leq-2
$$

see [CH09c, Lemma 4.8]. Further, the first set of equations in (3.2) implies that $c_{13}^{\rho_{1} \rho_{2}(a)}=0$, and hence $c_{23}^{\rho_{1} \rho_{2}(a)}<0$ by (1). Since $\sigma_{2}^{a}\left(2 \alpha_{1}+2 \alpha_{2}+\alpha_{3}\right)=2 \alpha_{1}+$ $\alpha_{3}-c_{23}^{a} \alpha_{2}$, the first set of equations in (3.2) and (4) imply that $k_{0}=2$. Similarly, Corollary 2.9 tells us that $k_{0}=2$ under the second set of conditions in (3.2) if and only if $c_{13}^{\rho_{2}(a)} \leq-2$.

It remains to consider the situation for

$$
c_{21}^{a}=-1, c_{23}^{a}=0, c_{13}^{\rho_{2}(a)}=-1,
$$

because, indeed, equation $c_{23}^{a}=0$ implies that $c_{23}^{\rho_{2}(a)}=0$ by $(\mathrm{C} 2)$, and hence $c_{13}^{\rho_{2}(a)}<0$ by (1). Assuming (3.3) we obtain that $\sigma_{2}^{a}\left(3 \alpha_{1}+2 \alpha_{2}+\alpha_{3}\right)=3 \alpha_{1}+\alpha_{2}+\alpha_{3}$, and hence (3) implies that $k_{0}=3$ if and only if the corresponding conditions in (5) are valid.

The rest follows by looking at $\sigma_{1} \sigma_{2}^{a}\left(4 \alpha_{1}+2 \alpha_{2}+\alpha_{3}\right)$; the details are left to the reader. The last claim holds since $c_{13}^{a}=0$ implies that $c_{23}^{a} \neq 0$ by (1). The assumption $\#\left(R_{+}^{a} \cap\left(\mathbb{Z} \alpha_{1}+\mathbb{Z} \alpha_{2}\right)\right) \geq 5$ is needed to exclude the case $c_{21}^{a}=-1$, $c_{12}^{\rho_{2}(a)}=-2, c_{21}^{\rho_{1} \rho_{2}(a)}=-1$, where $R_{+}^{a} \cap\left(\mathbb{Z} \alpha_{1}+\mathbb{Z} \alpha_{2}\right)=\left\{\alpha_{2}, \alpha_{1}+\alpha_{2}, 2 \alpha_{1}+\alpha_{2}, \alpha_{1}\right\}$, by using Proposition 2.6 and Corollary 2.9] see also the proof of [CH09c, Lemma 4.8].

Theorem 3.13. Let $\mathcal{C}$ be a Cartan scheme of rank three. Assume that $\mathcal{R}^{\mathrm{re}}$ is a finite irreducible root system of type $\mathcal{C}$. Then all entries of the Cartan matrices of $\mathcal{C}$ are greater than or equal to -7 . 
Proof. It can be assumed that $\mathcal{C}$ is connected. We prove the theorem indirectly. To do so we may assume that $a \in A$ such that $c_{12}^{a} \leq-8$. Then Proposition 2.6 implies that $\#\left(R_{+}^{a} \cap\left(\mathbb{Z} \alpha_{1}+\mathbb{Z} \alpha_{2}\right)\right) \geq 5$. By Lemma 3.12 there exists $k_{0} \in\{0,1,2,3,4\}$ such that $\alpha:=k_{0} \alpha_{1}+2 \alpha_{2}+\alpha_{3} \in R_{+}^{a}$ and $\alpha-\alpha_{1} \notin R^{a}$. By Lemma 3.2 and the choice of $k_{0}$ the set $\left\{\alpha, \alpha_{1}\right\}$ is a base for $V^{a}\left(\alpha, \alpha_{1}\right)$ at $a$. Corollary 2.5 implies that there exists a root $\gamma \in R^{a}$ such that $\left\{\alpha, \alpha_{1}, \gamma\right\}$ is a base for $\mathbb{Z}^{I}$ at $a$. Let $d \in A$, $w \in \operatorname{Hom}(a, d)$, and $i_{1}, i_{2}, i_{3} \in I$ such that $w(\alpha)=\alpha_{i_{1}}, w\left(\alpha_{1}\right)=\alpha_{i_{2}}, w(\gamma)=\alpha_{i_{3}}$.

Let $b=\rho_{1}(a)$. Again by Lemma 3.12 there exists $k_{1} \in\{0,1,2,3,4\}$ such that $\beta:=k_{1} \alpha_{1}+2 \alpha_{2}+\alpha_{3} \in R_{+}^{b}$. Thus

$$
R_{+}^{a} \ni \sigma_{1}^{b}(\beta)=\left(-k_{1}-2 c_{12}^{a}-c_{13}^{a}\right) \alpha_{1}+2 \alpha_{2}+\alpha_{3} .
$$

Further,

$$
-k_{1}-2 c_{12}^{a}-c_{13}^{a}-k_{0}>-c_{12}^{a}
$$

since $k_{0} \leq 2$ if $c_{13}^{a}=0$. Hence $\alpha_{i_{1}}+\left(1-c_{12}^{a}\right) \alpha_{i_{2}} \in R^{d}$, that is, $c_{i_{2} i_{1}}^{d}<c_{12}^{a} \leq-8$. We conclude that there exists no lower bound for the entries of the Cartan matrices of $\mathcal{C}$, which is a contradiction to the finiteness of $\mathcal{R}^{\mathrm{re}}(\mathcal{C})$. This proves the theorem.

Remark 3.14. The bound in the theorem is not sharp. After completing the classification one can check that the entries of the Cartan matrices of $\mathcal{C}$ are always at least -6 . The entry -6 appears for example in the Cartan scheme corresponding to the root system with number 53; see Corollary 2.9.

Proposition 3.15. Let $\mathcal{C}$ be an irreducible connected simply connected Cartan scheme of rank three. Assume that $\mathcal{R}^{\mathrm{re}}(\mathcal{C})$ is a finite root system of type $\mathcal{C}$. Let e be the number of vertices, $k$ the number of edges, and $f$ the number of (2-dimensional) faces of the object change diagram of $\mathcal{C}$. Then $e-k+f=2$.

Proof. The vertices of the object change diagram correspond to elements of $A$. Since $\mathcal{C}$ is connected and $\mathcal{R}^{\mathrm{re}}(\mathcal{C})$ is finite, the set $A$ is finite. Consider the equivalence relation on $I \times A$, where $(i, a)$ is equivalent to $(j, b)$ for some $i, j \in I$ and $a, b \in A$ if and only if $i=j$ and $b \in\left\{a, \rho_{i}(a)\right\}$. (This is also known as the pushout of $I \times A$ along the bijections id $: I \times A \rightarrow I \times A$ and $\rho: I \times A \rightarrow I \times A,(i, a) \mapsto$ $\left(i, \rho_{i}(a)\right)$.) Since $\mathcal{C}$ is simply connected, $\rho_{i}(a) \neq a$ for all $i \in I$ and $a \in A$. Then the edges of the object change diagram correspond to equivalence classes in $I \times A$. The faces of the object change diagram can be defined as equivalence classes of triples $(i, j, a) \in I \times I \times A \backslash\{(i, i, a) \mid i \in I, a \in A\}$, where $(i, j, a)$ and $\left(i^{\prime}, j^{\prime}, b\right)$ are equivalent for some $i, j, i^{\prime}, j^{\prime} \in I$ and $a, b \in A$ if and only if $\{i, j\}=\left\{i^{\prime}, j^{\prime}\right\}$ and $b \in\left\{\left(\rho_{j} \rho_{i}\right)^{m}(a), \rho_{i}\left(\rho_{j} \rho_{i}\right)^{m}(a) \mid m \in \mathbb{N}_{0}\right\}$. Since $\mathcal{C}$ is simply connected, (R4) implies that the face corresponding to a triple $(i, j, a)$ is a polygon with $2 m_{i, j}^{a}$ vertices.

For each face choose a triangulation by nonintersecting diagonals. Let $d$ be the total number of diagonals arising this way. Now consider the following twodimensional simplicial complex $C$ : The 0 -simplices are the objects. The 1-simplices are the edges and the chosen diagonals of the faces of the object change diagram. The 2 -simplices are the $f+d$ triangles. Clearly, each edge is contained in precisely two triangles. By [tD91, Ch. III, (3.3), 2,3] the geometric realization $X$ of $C$ is a closed 2-dimensional surface without boundary. The space $X$ is connected and compact.

Any two morphisms of $\mathcal{W}(\mathcal{C})$ with the same source and target are equal because $\mathcal{C}$ is simply connected. By [CH09c, Thm. 2.6] this equality follows from the Coxeter relations. A Coxeter relation means for the object change diagram that for the 


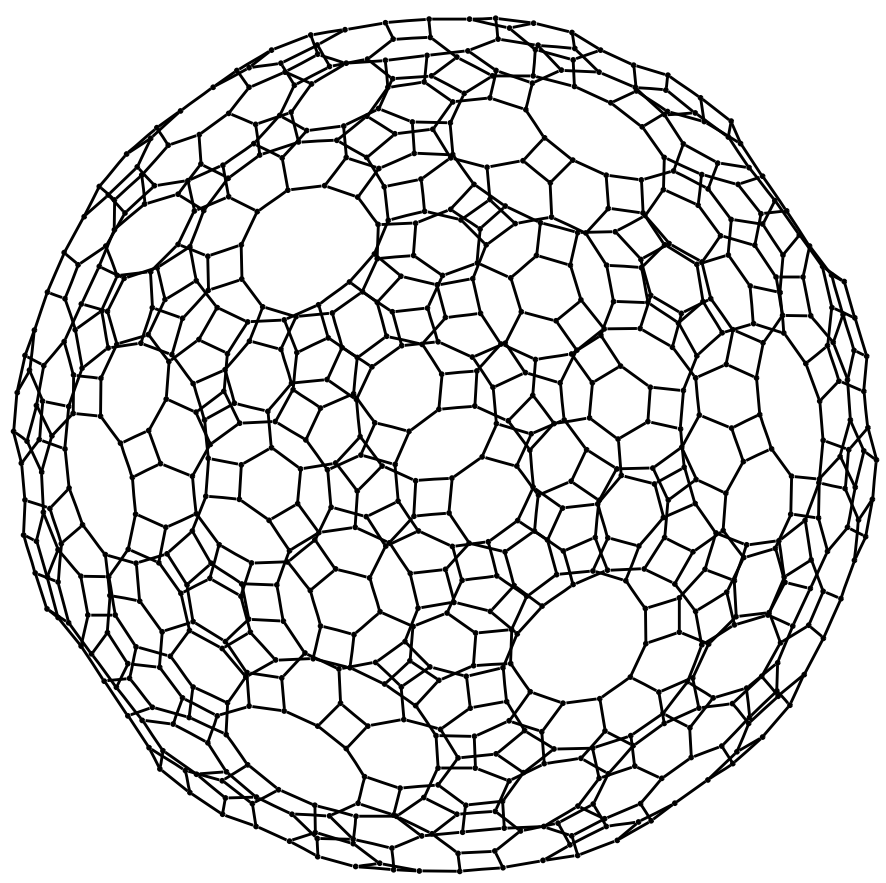

FiguRE 2. The object change diagram of the last root system of rank three

corresponding face and vertex the two paths along the sides of the face towards the opposite vertex yield the same morphism. But since this relation involves only one face which is simply connected, the corresponding paths are homotopic. Hence $X$ is simply connected and therefore homeomorphic to a two-dimensional sphere by tD91, Ch. III, Satz 6.9]. Its Euler characteristic is $2=e-(k+d)+(f+d)=$ $e-k+f$.

Remark 3.16. Assume that $\mathcal{C}$ is connected and simply connected, and let $a \in A$. Then any pair of opposite 2-dimensional faces of the object change diagram can be interpreted as a plane in $\mathbb{Z}^{I}$ containing at least two positive roots $\alpha, \beta \in R_{+}^{a}$. Indeed, let $b \in A$ and $i_{1}, i_{2} \in I$ with $i_{1} \neq i_{2}$. Since $\mathcal{C}$ is connected and simply connected, there exists a unique $w \in \operatorname{Hom}(a, b)$. Then $V^{a}\left(w^{-1}\left(\alpha_{i_{1}}\right), w^{-1}\left(\alpha_{i_{2}}\right)\right)$ is a plane in $\mathbb{Z}^{I}$ containing at least two positive roots. One can easily check that this plane is independent of the choice of the representative of the face determined by $\left(i_{1}, i_{2}, b\right) \in I \times I \times A$. Further, let $w_{0} \in \operatorname{Hom}(b, d)$, where $d \in A$, be the longest element in $\operatorname{Hom}(b, \mathcal{W}(\mathcal{C}))$. Let $j_{1}, j_{2} \in I$ such that $w_{0}\left(\alpha_{i_{n}}\right)=-\alpha_{j_{n}}$ for $n=1,2$. Then $\left(j_{1}, j_{2}, d\right)$ determines the plane

$$
V^{a}\left(\left(w_{0} w\right)^{-1}\left(\alpha_{j_{1}}\right),\left(w_{0} w\right)^{-1}\left(\alpha_{j_{2}}\right)\right)=V^{a}\left(w^{-1}\left(\alpha_{i_{1}}\right), w^{-1}\left(\alpha_{i_{2}}\right)\right) .
$$

This way we attached to any pair of (2-dimensional) opposite faces of the object change diagram a plane containing at least two positive roots. Let $<$ be a semigroup ordering on $\mathbb{Z}^{I}$ such that $0<\gamma$ for all $\gamma \in R_{+}^{a}$. Let $\alpha, \beta \in R_{+}^{a}$ with $\alpha \neq \beta$, and assume that $\alpha$ and $\beta$ are the smallest elements in $R_{+}^{a} \cap V^{a}(\alpha, \beta)$ with respect to $<$. Then $\{\alpha, \beta\}$ is a base for $V^{a}(\alpha, \beta)$ at $a$ by Lemma 3.3. By Corollary 2.5 there exists $b \in A$ and $w \in \operatorname{Hom}(a, b)$ such that $w(\alpha), w(\beta) \in R_{+}^{b}$ are simple roots. Hence 
any plane in $\mathbb{Z}^{I}$ containing at least two elements of $R_{+}^{a}$ can be obtained by the construction in the previous paragraph.

It remains to show that different pairs of opposite faces give rise to different planes. This follows from the fact that for any $b \in A$ and $i_{1}, i_{2} \in I$ with $i_{1} \neq i_{2}$ the conditions

$$
d \in A, u \in \operatorname{Hom}(b, d), j_{1}, j_{2} \in I, u\left(\alpha_{i_{1}}\right)=\alpha_{j_{1}}, u\left(\alpha_{i_{2}}\right)=\alpha_{j_{2}}
$$

have precisely two solutions: $u=\mathrm{id}_{b}$ on the one side, and $u=w_{0} w_{i_{1} i_{2}}$ on the other side, where $w_{i_{1} i_{2}}=\cdots \sigma_{i_{1}} \sigma_{i_{2}} \sigma_{i_{1}} \mathrm{id}_{b} \in \operatorname{Hom}(b, \mathcal{W}(\mathcal{C}))$ is the longest product of reflections $\sigma_{i_{1}}, \sigma_{i_{2}}$, and $w_{0}$ is an appropriate longest element of $\mathcal{W}(\mathcal{C})$. The latter follows from the fact that $u$ has to map the base $\left\{\alpha_{i_{1}}, \alpha_{i_{2}}, \alpha_{i_{3}}\right\}$ for $\mathbb{Z}^{I}$ at $b$, where $I=\left\{i_{1}, i_{2}, i_{3}\right\}$, to another base, and any base consisting of two simple roots can be extended precisely in two ways to a base of $\mathbb{Z}^{I}$ : by adding the third simple root or by adding a uniquely determined negative root.

It follows from the construction and by $\mathrm{CH09b}$, Lemma 6.4] that the faces corresponding to a plane $V^{a}(\alpha, \beta)$, where $\alpha, \beta \in R_{+}^{a}$ with $\alpha \neq \beta$, have as many edges as the cardinality of $V^{a}(\alpha, \beta) \cap R^{a}$ (or twice the cardinality of $V^{a}(\alpha, \beta) \cap R_{+}^{a}$ ).

Theorem 3.17. Let $\mathcal{C}$ be a connected simply connected Cartan scheme of rank three. Assume that $\mathcal{R}^{\mathrm{re}}(\mathcal{C})$ is a finite irreducible root system of type $\mathcal{C}$. Let $a \in A$ and let $M$ be the set of planes containing at least two elements of $R_{+}^{a}$. Then

$$
\sum_{V \in M} \#\left(V \cap R_{+}^{a}\right)=3(\# M-1) .
$$

Proof. Let $e, k, f$ be as in Proposition 3.15. Then $\# M=f / 2$ by Remark 3.16. For any vertex $b \in A$ there are three edges starting at $b$, and any edge is bounded by two vertices. Hence counting vertices in two different ways one obtains that $3 e=2 k$. Proposition 3.15 gives that $e-k+f=2$. Hence $2 k=3 e=3(2-f+k)$; that is, $k=3 f-6$.

Any plane $V$ corresponds to a face which is a polygon consisting of $2 \#\left(V \cap R_{+}^{a}\right)$ edges; see Remark 3.16. Summing up the edges twice over all planes (that is, summing up over all faces of the object change diagram), each edge is counted twice. Hence

$$
2 \sum_{V \in M} 2 \#\left(V \cap R_{+}^{a}\right)=2 k=2(3 f-6),
$$

which is the formula claimed in the theorem.

Corollary 3.18. Let $\mathcal{C}$ be a connected simply connected Cartan scheme of rank three. Assume that $\mathcal{R}^{\mathrm{re}}(\mathcal{C})$ is a finite irreducible root system of type $\mathcal{C}$. Then there exists an object $a \in A$ and $\alpha, \beta, \gamma \in R_{+}^{a}$ such that $\{\alpha, \beta, \gamma\}=\left\{\alpha_{1}, \alpha_{2}, \alpha_{3}\right\}$ and

$$
\#\left(V^{a}(\alpha, \beta) \cap R_{+}^{a}\right)=2, \quad \#\left(V^{a}(\alpha, \gamma) \cap R_{+}^{a}\right)=3 .
$$

Further, $\alpha+\gamma, \beta+\gamma, \alpha+\beta+\gamma \in R_{+}^{a}$.

Proof. Let $M$ be as in Theorem 3.17. Let $a$ be any object and assume $\#\left(V \cap R_{+}^{a}\right)>$ 2 for all $V \in M$. Then $\sum_{V \in M} \#\left(V \cap R_{+}^{a}\right) \geq 3 \# M$, contradicting Theorem 3.17 Hence for all objects $a$ there exists a plane $V$ with $\#\left(V \cap R_{+}^{a}\right)=2$. Now consider the object change diagram and count the number of faces: Let $2 q_{i}$ be the number of faces with $2 i$ edges. Then Theorem 3.17 translates to

$$
\sum_{i \geq 2} i q_{i}=-3+3 \sum_{i \geq 2} q_{i} .
$$


Assume that there exists no object adjacent to a square and a hexagon. Since $\mathcal{R}^{\mathrm{re}}(\mathcal{C})$ is irreducible, no two squares have a common edge; see Lemma 3.12(1). Look at the edges ending in vertices of squares, and count each edge once for both polygons adjacent to it. One checks that there are at least twice as many edges adjacent to a polygon with at least 8 vertices as edges of squares. This gives that

$$
\sum_{i \geq 4} 2 i \cdot 2 q_{i} \geq 2 \cdot 4 \cdot 2 q_{2} \text {. }
$$

By equation (3.5) we then have $-3+3 \sum_{i \geq 2} q_{i} \geq 4 q_{2}+2 q_{2}+3 q_{3}$, that is, $q_{2}<$ $\sum_{i \geq 4} q_{i}$. But then in average each face has more than 6 edges which contradicts Theorem 3.17. Hence there is an object $a$ such that there exist $\alpha, \beta, \gamma \in R_{+}^{a}$ as above satisfying equation (3.4). We have $\alpha+\gamma, \beta+\gamma, \alpha+\beta+\gamma \in R_{+}^{a}$ by Lemma3.12(1),(2) and Corollary 2.9 .

\section{THE CLASSIFICATION}

In this section we explain the classification of connected simply connected Cartan schemes of rank three such that $\mathcal{R}^{\text {re }}(\mathcal{C})$ is a finite irreducible root system. We formulate the main result in Theorem 4.1. The proof of Theorem 4.1 is performed using computer calculations based on results of the previous sections. Our algorithm described below is sufficiently powerful: The implementation in $C$ terminates within a few hours on a usual computer and is available at

http://www . mathematik. uni-kl.de/ cuntz/download/wgr3.c.

Removing any of the theorems, the calculations would take at least several weeks.

Theorem 4.1. (1) Let $\mathcal{C}$ be a connected Cartan scheme of rank three with $I=$ $\{1,2,3\}$. Assume that $\mathcal{R}^{\mathrm{re}}(\mathcal{C})$ is a finite irreducible root system of type $\mathcal{C}$. Then there exists an object $a \in A$ and a linear map $\tau \in \operatorname{Aut}\left(\mathbb{Z}^{I}\right)$ such that $\tau\left(\alpha_{i}\right) \in\left\{\alpha_{1}, \alpha_{2}, \alpha_{3}\right\}$ for all $i \in I$ and $\tau\left(R_{+}^{a}\right)$ is one of the sets listed in Appendix $\mathrm{A}$. Moreover, $\tau\left(R_{+}^{a}\right)$ with this property is uniquely determined.

(2) Let $R$ be one of the 55 subsets of $\mathbb{Z}^{3}$ appearing in Appendix A, There exists up to equivalence a unique connected simply connected Cartan scheme $\mathcal{C}\left(I, A,\left(\rho_{i}\right)_{i \in I}\right.$, $\left.\left(C^{a}\right)_{a \in A}\right)$ such that $R \cup-R$ is the set of real roots $R^{a}$ in an object $a \in A$. Moreover $\mathcal{R}^{\mathrm{re}}(\mathcal{C})$ is a finite irreducible root system of type $\mathcal{C}$.

Remark 4.2. Theorem 4.1 is a classification of connected simply connected Cartan schemes $\mathcal{C}$, up to equivalence in the sense of [CH09c, Def. 2.1], such that $\mathcal{R}^{\mathrm{re}}(\mathcal{C})$ is a finite root system of type $\mathcal{C}$. To obtain a classification of connected Cartan schemes such that $\mathcal{R}^{\mathrm{re}}(\mathcal{C})$ is a finite root system, one additionally has to classify quotients (inverse of coverings) of our simply connected Cartan schemes, which amounts to classifying all subgroups of $\operatorname{Hom}(a)$ in Table 1 . We do not claim that Weyl groupoids of nonequivalent Cartan schemes are nonisomorphic as groupoids. It should also be stressed that the list of root systems in the appendix contains for each Cartan scheme the root system of precisely one object. We explain in the appendix in which sense this root system is canonical.

Let $<$ be the lexicographic ordering on $\mathbb{Z}^{3}$ such that $\alpha_{3}<\alpha_{2}<\alpha_{1}$. Then $\alpha>0$ for any $\alpha \in \mathbb{N}_{0}^{3} \backslash\{0\}$.

Let $\mathcal{C}$ be a connected Cartan scheme with $I=\{1,2,3\}$. Assume that $\mathcal{R}^{\text {re }}(\mathcal{C})$ is a finite irreducible root system of type $\mathcal{C}$. Let $a \in A$. By Theorem 2.10 we may construct $R_{+}^{a}$ inductively by starting with $R_{+}^{a}=\left\{\alpha_{3}, \alpha_{2}, \alpha_{1}\right\}$, and appending in 
each step a sum of a pair of positive roots which is greater than all roots in $R_{+}^{a}$ we already have. During this process, we keep track of all planes containing at least two positive roots, and the positive roots on them. Lemma 3.3 implies that for any known root $\alpha$ and new root $\beta$ either $V^{a}(\alpha, \beta)$ contains no other known positive roots, or $\beta$ is not part of the unique base for $V^{a}(\alpha, \beta)$ at $a$ consisting of positive roots. In the first case the roots $\alpha, \beta$ generate a new plane. It can happen that $\operatorname{Vol}_{2}(\alpha, \beta)>1$, and then $\{\alpha, \beta\}$ is not a base for $V^{a}(\alpha, \beta)$ at $a$, but we don't care about that. In the second case the known roots in $V^{a}(\alpha, \beta) \cap R_{+}^{a}$ together with $\beta$ have to form an $\mathcal{F}$-sequence by Proposition 2.6 .

Sometimes, by some theorem (see the details below) we know that it is not possible to add more positive roots to a plane. Then we can mark it as "finished".

Observe that to obtain a finite number of root systems as output, we have to ensure that we compute only irreducible systems since there are infinitely many inequivalent reducible root systems of rank two. Hence starting with $\left\{\alpha_{3}, \alpha_{2}, \alpha_{1}\right\}$ will not work. However, by Corollary 3.18, starting with $\left\{\alpha_{3}, \alpha_{2}, \alpha_{2}+\alpha_{3}, \alpha_{1}, \alpha_{1}+\right.$ $\left.\alpha_{2}, \alpha_{1}+\alpha_{2}+\alpha_{3}\right\}$ will still yield at least one root system for each desired Cartan scheme (notice that any roots one would want to add are lexicographically greater).

In this section, we will call root system fragment (or $r s f$ ) the following set of data associated to a set of positive roots $R$ in construction:

- normal vectors for the planes with at least two positive roots

- labels of positive roots on these planes

- Cartan entries corresponding to the root systems of the planes

- an array of flags for finished planes

- the sum $s_{R}$ of $\#(V \cap R)$ over all planes $V$ with at least two positive roots; see Theorem 3.17

- for each root $r \in R$ the list of planes it belongs to.

These data can be obtained directly from $R$, but the calculation is faster if we continuously update them.

We divide the algorithm into three parts. The main part is Algorithm 4.4; see below.

The first part updates a root system fragment to a new root and uses Theorems 3.10 and 3.13 to possibly refuse doing so:

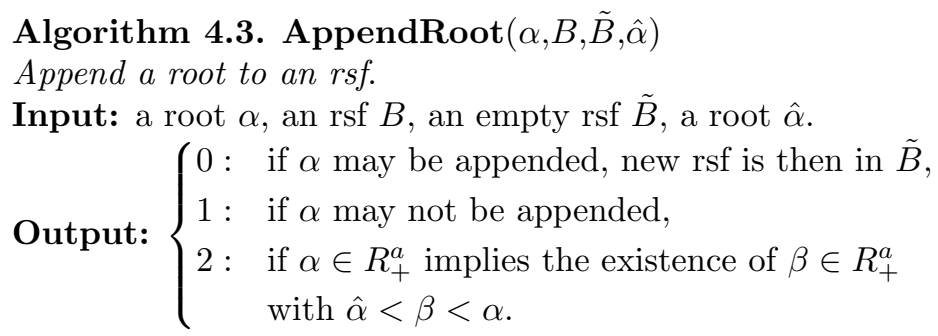

1. Let $r$ be the number of planes containing at least two elements of $R$. For documentation purposes let $V_{1}, \ldots, V_{r}$ denote these planes. For any $i \in$ $\{1, \ldots, r\}$ let $v_{i}$ be a normal vector for $V_{i}$, and let $R_{i}$ be the $\mathcal{F}$-sequence of $V_{i} \cap R$. Set $i \leftarrow 1, g \leftarrow 1, c \leftarrow[], p \leftarrow[], d \leftarrow\{\}$. During the algorithm, $c$ will be an ordered subset of $\{1, \ldots, r\}, p$ a corresponding list of "positions", and $d$ a subset of $R$. 
2. If $i \leq r$ and $g \neq 0$, then compute the scalar product $g:=\left(\alpha, v_{i}\right)$. (Then $g=$ $\operatorname{det}\left(\alpha, \gamma_{1}, \gamma_{2}\right)= \pm \operatorname{Vol}_{3}\left(\alpha, \gamma_{1}, \gamma_{2}\right)$, where $\left\{\gamma_{1}, \gamma_{2}\right\}$ is the basis of $V_{i}$ consisting of positive roots.) Otherwise go to Step 6 .

3. If $g=0$, then do the following: If $V_{i}$ is not yet finished, then check if $\alpha$ extends $R_{i}$ to a new $\mathcal{F}$-sequence. If yes, add the roots of $R_{i}$ to $d$, append $i$ to $c$, append the position of the insertion of $\alpha$ in $R_{i}$ to $p$, let $g \leftarrow 1$, and go to Step 5 .

4. If $g^{2}=1$, then use Corollary 3.11 Let $\gamma_{1}$ and $\gamma_{2}$ be the beginning and the end of the $\mathcal{F}$-sequence $R_{i}$, respectively. (Then $\left\{\gamma_{1}, \gamma_{2}\right\}$ is a base for $V_{i}$ at a.) Let $\delta_{1} \leftarrow \alpha-\gamma_{1}, \delta_{2} \leftarrow \alpha-\gamma_{2}$. If $\delta_{1}, \delta_{2} \notin R$, then return 1 if $\delta_{1}, \delta_{2} \leq \hat{\alpha}$ and return 2 otherwise.

5. Set $i \leftarrow i+1$ and go to Step 2 .

6. If there is no objection appending $\alpha$ so far, i.e. $g \neq 0$, then copy $B$ to $\tilde{B}$ and include $\alpha$ into $\tilde{B}$ : use $c, p$ to extend existing $\mathcal{F}$-sequences, and use (the complement of) $d$ to create new planes. Finally, apply Theorem 3.13, If there is a Cartan entry less than -7 , then return 1, else return 0. If $g=0$, then return 2 .

The second part looks for small roots which we must include in any case. The function is based on Proposition 3.6. This is a strong restriction during the process.

Algorithm 4.4. RequiredRoot $(R, B, \hat{\alpha})$

Find a smallest required root under the assumption that all roots $\leq \hat{\alpha}$ are known.

Input: $R$ a set of roots, $B$ an rsf for $R, \hat{\alpha}$ a root.

Output: $\begin{cases}0 & \text { if we cannot determine such a root, } \\ 1, \varepsilon & \text { if we have found a small missing root } \varepsilon \text { with } \varepsilon>\hat{\alpha}, \\ 2 & \text { if the given configuration is impossible. }\end{cases}$

1. Initialize the return value $f \leftarrow 0$.

2. We use the same notation as in Algorithm 4.2, Step 1. For all $\gamma_{1}$ in $R$ and all $(j, k) \in\{1, \ldots, r\} \times\{1, \ldots, r\}$ such that $j \neq k, \gamma_{1} \in R_{j} \cap R_{k}$, and both $R_{j}, R_{k}$ contain two elements, let $\gamma_{2}, \gamma_{3} \in R$ such that $R_{j}=\left\{\gamma_{1}, \gamma_{2}\right\}$, $R_{k}=\left\{\gamma_{1}, \gamma_{3}\right\}$. If $\operatorname{Vol}_{3}\left(\gamma_{1}, \gamma_{2}, \gamma_{3}\right)=1$, then do Steps 3 to 6 .

3. $\xi_{2} \leftarrow \gamma_{1}+\gamma_{2}, \xi_{3} \leftarrow \gamma_{1}+\gamma_{3}$.

4. If $\hat{\alpha} \geq \xi_{2}$ : If $\hat{\alpha} \geq \xi_{3}$ or plane $V_{k}$ is already finished, then return 2. If $f=0$ or $\varepsilon>\xi_{3}$, then $\varepsilon \leftarrow \xi_{3}, f \leftarrow 1$. Go to Step 2 and continue the loop.

5. If $\hat{\alpha} \geq \xi_{3}$ : If plane $V_{j}$ is already finished, then return 2. If $f=0$ or $\varepsilon>\xi_{2}$, then $\varepsilon \leftarrow \xi_{2}, f \leftarrow 1$.

6. Go to Step 2 and continue the loop.

7. Return $f, \varepsilon$.

Finally, we resursively add roots to a set, update the rsf and include required roots:

Algorithm 4.5. CompleteRootSystem $(R, B, \hat{\alpha}, u, \beta)$
Collects potential new roots, appends them and calls itself again.

Input: $R$ a set of roots, $B$ an rsf for $R, \hat{\alpha}$ a lower bound for new roots, $u$ a flag, $\beta$ a vector which is necessarily a root if $u=$ True.

Output: Root systems containing $R$. 
1. Check Theorem 3.17, If $s_{R}=3(r-1)$, where $r$ is the number of planes containing at least two positive roots, then output $R$ (and continue). We have found a potential root system.

2. If we have no required root yet, i.e. $u=$ False, then $f, \varepsilon:=\operatorname{RequiredRoot}(R, B, \hat{\alpha})$. If $f=1$, then we have found a required root; we call CompleteRootSystem $(R, B, \hat{\alpha}, \operatorname{True}, \varepsilon)$ and terminate. If $f=$ 2 , then terminate.

3. Potential new roots will be collected in $Y \leftarrow\{\} ; \tilde{B}$ will be the new rsf.

4. For all planes $V_{i}$ of $B$ which are not finished, do Steps 5 to 7 .

5. $\nu \leftarrow 0$.

6. For $\zeta$ in the set of roots that may be added to the plane $V_{i}$ such that $\zeta>\hat{\alpha}$, do the following:

- set $\nu \leftarrow \nu+1$.

- If $\zeta \notin Y$, then $Y \leftarrow Y \cup\{\zeta\}$. If moreover $u=$ False or $\beta>\zeta$, then $-y \leftarrow \operatorname{AppendRoot}(\zeta, B, \tilde{B}, \hat{\alpha})$

- if $y=0$, then CompleteRootSystem $(R \cup\{\zeta\}, \tilde{B}, \zeta, u, \beta)$.

- if $y=1$, then $\nu \leftarrow \nu-1$.

7. If $\nu=0$, then mark $V_{i}$ as finished in $\tilde{B}$.

8. if $u=$ True and $\operatorname{AppendRoot}(\beta, B, \tilde{B}, \hat{\alpha})=0$, then call

CompleteRootSystem $(R \cup\{\beta\}, \tilde{B}, \beta$, False, $\beta)$.

Terminate the function call.

Note that we only used necessary conditions for root systems, so after the computation we still need to check which of the sets are indeed root systems. A short program in MAGMA confirms that Algorithm 4.4 yields only root systems, for instance using this algorithm:

\section{Algorithm 4.6. RootSystemsForAllObjects $(R)$}

Returns the root systems for all objects if $R=R_{+}^{a}$ determines a Cartan scheme $\mathcal{C}$ such that $\mathcal{R}^{\mathrm{re}}(\mathcal{C})$ is an irreducible root system.

Input: $R$ the set of positive roots at one object.

Output: the set of root systems at all objects, or \{\} if $R$ does not yield a Cartan scheme as desired.

1. $N \leftarrow[R], M \leftarrow\{\}$.

2. While $|N|>0$, do Steps 3 to 5 .

3. Let $F$ be the last element of $N$. Remove $F$ from $N$ and include it to $M$.

4. Let $C$ be the Cartan matrix of $F$. Compute the three simple reflections given by $C$.

5. For each simple reflection $s$, do:

- Compute $G:=\{s(v) \mid v \in F\}$. If an element of $G$ has positive and negative coefficients, then return \{\} . Otherwise multiply the negative roots of $G$ by -1 .

- If $G \notin M$, then append $G$ to $N$.

6. Return $M$.

Remark 4.7. We list all 55 root systems in Appendix A. It is also interesting to summarize some of the invariants, which is done in Table 1 . Let $\mathcal{O}=\left\{R^{a} \mid a \in A\right\}$ denote the set of root systems in the objects of the Cartan scheme. By identifying 
objects with the same root system one obtains a quotient Cartan scheme of the simply connected Cartan scheme of the classification (see [CH09b, Def. 3.1] for the definition of coverings). This quotient has the minimal number of objects with respect to all quotients of the Cartan scheme. In the fifth column we give the automorphism group of one (equivalently, any) object of this quotient. The last column gives the multiplicities of planes; for example, $3^{7}$ means that there are 7 different planes containing precisely 3 positive roots.

TABLE 1. Invariants of irreducible root systems of rank three; see Remark 4.7

\begin{tabular}{|c|c|c|c|c|c|}
\hline $\mathrm{Nr}$. & $\mid R_{+}^{a}$ & $|\mathcal{O}|$ & $\overline{A \mid}$ & $\operatorname{Hom}(a)$ & planes \\
\hline 1 & 6 & 1 & 24 & $A_{3}$ & $2^{3}, 3^{4}$ \\
\hline 2 & 7 & 4 & 32 & $A_{1} \times A_{1} \times A_{1}$ & $2^{3}, 3^{6}$ \\
\hline 3 & 8 & 5 & 40 & $B_{2}$ & $2^{4}, 3^{6}, 4^{1}$ \\
\hline 4 & 9 & 1 & 48 & $B_{3}$ & $2^{6}, 3^{4}, 4^{3}$ \\
\hline 5 & 9 & 1 & 48 & $B_{3}$ & $2^{6}, 3^{4}, 4^{3}$ \\
\hline 6 & 10 & 5 & 60 & $A_{1} \times A_{2}$ & $2^{6}, 3^{7}, 4^{3}$ \\
\hline 7 & 10 & 10 & 60 & $A_{2}$ & $2^{6}, 3^{7}, 4^{3}$ \\
\hline 8 & 11 & 9 & 72 & $A_{1} \times A_{1} \times A_{1}$ & $2^{7}, 3^{8}, 4^{4}$ \\
\hline 9 & 12 & 21 & 84 & $A_{1} \times A_{1}$ & $2^{8}, 3^{10}, 4^{3}, 5^{1}$ \\
\hline 10 & 12 & 14 & 84 & $A_{2}$ & $2^{9}, 3^{7}, 4^{6}$ \\
\hline 11 & 13 & 4 & 96 & $G_{2} \times A_{1}$ & $2^{9}, 3^{12}, 4^{3}, 6^{1}$ \\
\hline 12 & 13 & 12 & 96 & $A_{1} \times A_{1} \times A_{1}$ & $2^{10}, 3^{10}, 4^{3}, 5^{2}$ \\
\hline 13 & 13 & 2 & 96 & $B_{3}$ & $2^{12}, 3^{4}, 4^{9}$ \\
\hline 14 & 13 & 2 & 96 & $B_{3}$ & $2^{12}, 3^{4}, 4^{9}$ \\
\hline 15 & 14 & 56 & 112 & $A_{1}$ & $2^{11}, 3^{12}, 4^{4}, 5^{2}$ \\
\hline 16 & 15 & 16 & 128 & $A_{1} \times A_{1} \times A_{1}$ & $2^{13}, 3^{12}, 4^{6}, 5^{2}$ \\
\hline 17 & 16 & 36 & 144 & $A_{1} \times A_{1}$ & $2^{14}, 3^{15}, 4^{6}, 5^{1}, 6^{1}$ \\
\hline 18 & 16 & 24 & 144 & $A_{2}$ & $2^{15}, 3^{13}, 4^{6}, 5^{3}$ \\
\hline 19 & 17 & 10 & 160 & $B_{2} \times A_{1}$ & $2^{16}, 3^{16}, 4^{7}, 6^{2}$ \\
\hline 20 & 17 & 10 & 160 & $B_{2} \times A_{1}$ & $2^{16}, 3^{16}, 4^{7}, 6^{2}$ \\
\hline 21 & 17 & 10 & 160 & $B_{2} \times A_{1}$ & $2^{18}, 3^{12}, 4^{7}, 5^{4}$ \\
\hline 22 & 18 & 30 & 180 & $A_{2}$ & $2^{18}, 3^{18}, 4^{6}, 5^{3}, 6^{1}$ \\
\hline 23 & 18 & 90 & 180 & $A_{1}$ & $2^{19}, 3^{16}, 4^{6}, 5^{5}$ \\
\hline 24 & 19 & 25 & 200 & $A_{1} \times A_{1} \times A_{1}$ & $2^{20}, 3^{20}, 4^{6}, 5^{4}, 6^{1}$ \\
\hline 25 & 19 & 8 & 192 & $G_{2} \times A_{1}$ & $2^{21}, 3^{18}, 4^{6}, 6^{4}$ \\
\hline 26 & 19 & 50 & 200 & $A_{1} \times A_{1}$ & $2^{20}, 3^{20}, 4^{6}, 5^{4}, 6^{1}$, \\
\hline 27 & 19 & 25 & 200 & $A_{1} \times A_{1} \times A_{1}$ & $2^{20}, 3^{20}, 4^{6}, 5^{4}, 6^{1}$ \\
\hline 28 & 19 & 8 & 192 & $G_{2} \times A_{1}$ & $2^{24}, 3^{12}, 4^{6}, 5^{6}, 6^{1}$ \\
\hline 29 & 20 & 27 & 216 & $B_{2}$ & $2^{20}, 3^{26}, 4^{4}, 5^{4}, 8^{1}$ \\
\hline 30 & 20 & 110 & 220 & $A_{1}$ & $2^{21}, 3^{24}, 4^{6}, 5^{4}, 7^{1}$ \\
\hline 31 & 20 & 110 & 220 & $A_{1}$ & $2^{23}, 3^{20}, 4^{7}, 5^{5}, 6^{1}$ \\
\hline 32 & 21 & 15 & 240 & $B_{2} \times A_{1}$ & $2^{22}, 3^{28}, 4^{6}, 5^{4}, 8^{1}$ \\
\hline 33 & 21 & 30 & 240 & $A_{1} \times A_{1} \times A_{1}$ & $2^{26}, 3^{20}, 4^{9}, 5^{4}, 6^{2}$ \\
\hline 34 & 21 & 5 & 240 & $B_{3}$ & $2^{24}, 3^{24}, 4^{9}, 6^{4}$ \\
\hline 35 & 22 & 44 & 264 & $A_{2}$ & $2^{27}, 3^{25}, 4^{9}, 5^{3}, 6^{3}$ \\
\hline 36 & 25 & 42 & 336 & $A_{1} \times A_{1} \times A_{1}$ & $2^{33}, 3^{34}, 4^{12}, 5^{2}, 6^{3}, 8^{1}$, \\
\hline 37 & 25 & 14 & 336 & $G_{2} \times A_{1}$ & $2^{36}, 3^{30}, 4^{9}, 5^{6}, 6^{4}$ \\
\hline 38 & 25 & 28 & 336 & $A_{1} \times A_{2}$ & $2^{36}, 3^{30}, 4^{9}, 5^{6}, 6^{4}$ \\
\hline
\end{tabular}




\begin{tabular}{|llllll|}
\hline Nr. & $\left|R_{+}^{a}\right|$ & $|\mathcal{O}|$ & $|A|$ & $\operatorname{Hom}(a)$ & planes \\
\hline 39 & 25 & 7 & 336 & $B_{3}$ & $2^{36}, 3^{28}, 4^{15}, 6^{6}$, \\
40 & 26 & 182 & 364 & $A_{1}$ & $2^{35}, 3^{39}, 4^{10}, 5^{4}, 6^{3}, 8^{1}$, \\
41 & 26 & 182 & 364 & $A_{1}$ & $2^{37}, 3^{36}, 4^{9}, 5^{6}, 6^{3}, 7^{1}$, \\
42 & 27 & 49 & 392 & $A_{1} \times A_{1} \times A_{1}$ & $2^{38}, 3^{42}, 4^{9}, 5^{6}, 6^{3}, 8^{1}$, \\
43 & 27 & 98 & 392 & $A_{1} \times A_{1}$ & $2^{39}, 3^{40}, 4^{10}, 5^{6}, 6^{2}, 7^{2}$, \\
44 & 27 & 98 & 392 & $A_{1} \times A_{1}$ & $2^{39}, 3^{40}, 4^{10}, 5^{6}, 6^{2}, 7^{2}$, \\
45 & 28 & 420 & 420 & 1 & $2^{41}, 3^{44}, 4^{11}, 5^{6}, 6^{2}, 7^{1}, 8^{1}$, \\
46 & 28 & 210 & 420 & $A_{1}$ & $2^{42}, 3^{42}, 4^{12}, 5^{6}, 6^{1}, 7^{3}$, \\
47 & 28 & 70 & 420 & $A_{2}$ & $2^{42}, 3^{42}, 4^{12}, 5^{6}, 6^{1}, 7^{3}$, \\
48 & 29 & 56 & 448 & $A_{1} \times A_{1} \times A_{1}$ & $2^{44}, 3^{46}, 4^{13}, 5^{6}, 6^{2}, 8^{2}$, \\
49 & 29 & 112 & 448 & $A_{1} \times A_{1}$ & $2^{45}, 3^{44}, 4^{14}, 5^{6}, 6^{1}, 7^{2}, 8^{1}$, \\
50 & 29 & 112 & 448 & $A_{1} \times A_{1}$ & $2^{45}, 3^{44}, 4^{14}, 5^{6}, 6^{1}, 7^{2}, 8^{1}$, \\
51 & 30 & 238 & 476 & $A_{1}$ & $2^{49}, 3^{44}, 4^{17}, 5^{6}, 6^{1}, 7^{1}, 8^{2}$, \\
52 & 31 & 21 & 504 & $G_{2} \times A_{1}$ & $2^{54}, 3^{42}, 4^{21}, 5^{6}, 6^{1}, 8^{3}$, \\
53 & 31 & 21 & 504 & $G_{2} \times A_{1}$ & $2^{54}, 3^{42}, 4^{21}, 5^{6}, 6^{1}, 8^{3}$, \\
54 & 34 & 102 & 612 & $A_{2}$ & $2^{60}, 3^{63}, 4^{18}, 5^{6}, 6^{4}, 8^{3}$, \\
55 & 37 & 15 & 720 & $B_{3}$ & $2^{72}, 3^{72}, 4^{24}, 6^{10}, 8^{3}$, \\
\hline
\end{tabular}

At first sight, one is tempted to look for a formula for the number of objects in the universal covering depending on the number of roots. There is an obvious one: consider the coefficients of $4 /\left((1-x)^{2}\left(1-x^{4}\right)\right)$. However, there are exceptions, for example nr. 29 with 20 positive roots and 216 objects (instead of 220 ).

Rank 3 Nichols algebras of diagonal type with a finite irreducible arithmetic root system are classified in [Hec05, Table 2]. In Table 2 we identify the Weyl groupoids of these Nichols algebras.

TABLE 2. Weyl groupoids of rank three Nichols algebras of diagonal type

\begin{tabular}{|l|lllllllll|}
\hline row in [Hec05, Table 2] & 1 & 2 & 3 & 4 & 5 & 6 & 7 & 8 & 9 \\
Weyl groupoid & 1 & 5 & 4 & 1 & 5 & 3 & 11 & 1 & 2 \\
\hline row in [Hec05, Table 2] & 10 & 11 & 12 & 13 & 14 & 15 & 16 & 17 & 18 \\
Weyl groupoid & 2 & 2 & 5 & 13 & 5 & 6 & 7 & 8 & 14 \\
\hline
\end{tabular}

Appendix A. Irreducible root systems of Rank three

We give the roots in a multiplicative notation to save space: The word $1^{x} 2^{y} 3^{z}$ corresponds to $x \alpha_{3}+y \alpha_{2}+z \alpha_{1}$.

Notice that we have chosen a "canonical" object for each groupoid. Write $\pi\left(R_{+}^{a}\right)$ for the set $R_{+}^{a}$, where the coordinates are permuted via $\pi \in S_{3}$. Then the set listed below is the minimum of $\left\{\pi\left(R_{+}^{a}\right) \mid a \in A, \pi \in S_{3}\right\}$ with respect to the lexicographical ordering on the sorted sequences of roots.

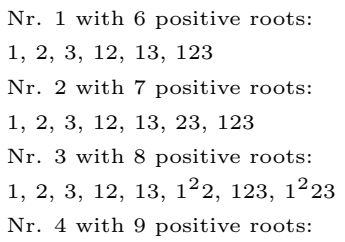


$1,2,3,12,13,1^{2} 2,123,1^{2} 23,1^{2} 23^{2}$

Nr. 5 with 9 positive roots:

$1,2,3,12,23,1^{2} 2,123,1^{2} 23,1^{2} 2^{2} 3$

Nr. 6 with 10 positive roots:

$1,2,3,12,13,1^{2} 2,1^{2} 3,123,1^{2} 23,1^{3} 23$

Nr. 7 with 10 positive roots:

$1,2,3,12,13,23,1^{2} 2,123,1^{2} 23,1^{2} 2^{2} 3$

Nr. 8 with 11 positive roots:

$1,2,3,12,13,1^{2} 2,1^{2} 3,123,1^{2} 23,1^{3} 23,1^{3} 2^{2} 3$

Nr. 9 with 12 positive roots:

$1,2,3,12,13,1^{2} 2,123,1^{3} 2,1^{2} 23,1^{3} 23,1^{3} 2^{2} 3,1^{4} 2^{2} 3$

Nr. 10 with 12 positive roots:

$1,2,3,12,13,1^{2} 2,1^{2} 3,123,1^{2} 23,1^{3} 23,1^{2} 2^{2} 3,1^{3} 2^{2} 3$

Nr. 11 with 13 positive roots:

$1,2,3,12,13,1^{2} 2,123,1^{3} 2,1^{2} 23,1^{3} 2^{2}, 1^{3} 23,1^{3} 2^{2} 3,1^{4} 2^{2} 3$

Nr. 12 with 13 positive roots:

$1,2,3,12,13,1^{2} 2,1^{2} 3,123,1^{3} 2,1^{2} 23,1^{3} 23,1^{4} 23,1^{4} 2^{2} 3$

Nr. 13 with 13 positive roots:

$1,2,3,12,13,1^{2} 2,1^{2} 3,123,1^{2} 23,1^{3} 23,1^{2} 2^{2} 3,1^{3} 2^{2} 3,1^{4} 2^{2} 3$

Nr. 14 with 13 positive roots:

$1,2,3,12,13,1^{2} 2,123,13^{2}, 1^{2} 23,123^{2}, 1^{2} 23^{2}, 1^{3} 23^{2}, 1^{3} 2^{2} 3^{2}$

Nr. 15 with 14 positive roots:

$1,2,3,12,13,1^{2} 2,1^{2} 3,123,1^{3} 2,1^{2} 23,1^{3} 23,1^{4} 23,1^{3} 2^{2} 3,1^{4} 2^{2} 3$

Nr. 16 with 15 positive roots:

$1,2,3,12,13,1^{2} 2,1^{2} 3,123,1^{3} 2,1^{2} 23,1^{3} 23,1^{4} 23,1^{3} 2^{2} 3,1^{4} 2^{2} 3,1^{5} 2^{2} 3$

Nr. 17 with 16 positive roots:

$1,2,3,12,13,1^{2} 2,1^{2} 3,123,1^{3} 2,1^{2} 23,1^{3} 2^{2}, 1^{3} 23,1^{4} 23,1^{3} 2^{2} 3,1^{4} 2^{2} 3,1^{5} 2^{2} 3$

Nr. 18 with 16 positive roots:

$1,2,3,12,23,1^{2} 2,123,1^{3} 2,1^{2} 23,12^{2} 3,1^{3} 23,1^{2} 2^{2} 3,1^{3} 2^{2} 3,1^{4} 2^{2} 3,1^{4} 2^{3} 3,1^{4} 2^{3} 3^{2}$

Nr. 19 with 17 positive roots:

$1,2,3,12,13,1^{2} 2,1^{2} 3,123,1^{3} 2,1^{2} 23,1^{4} 2,1^{3} 23,1^{4} 23,1^{5} 23,1^{4} 2^{2} 3,1^{5} 2^{2} 3,1^{6} 2^{2} 3$

Nr. 20 with 17 positive roots:

$1,2,3,12,13,1^{2} 2,1^{2} 3,123,1^{3} 2,1^{2} 23,1^{3} 2^{2}, 1^{3} 23,1^{4} 23,1^{3} 2^{2} 3,1^{4} 2^{2} 3,1^{5} 2^{2} 3,1^{5} 2^{2} 3^{2}$

Nr. 21 with 17 positive roots:

$1,2,3,12,13,1^{2} 2,123,1^{3} 2,1^{2} 23,1^{3} 23,1^{2} 2^{2} 3,1^{3} 2^{2} 3,1^{4} 2^{2} 3,1^{5} 2^{2} 3,1^{5} 2^{3} 3,1^{5} 2^{3} 3^{2}, 1^{6} 2^{3} 3^{2}$

Nr. 22 with 18 positive roots:

$1,2,3,12,13,1^{2} 2,1^{2} 3,123,1^{3} 2,1^{2} 23,1^{3} 2^{2}, 1^{3} 23,1^{4} 23,1^{3} 2^{2} 3,1^{4} 2^{2} 3,1^{5} 2^{2} 3,1^{5} 2^{3} 3,1^{6} 2^{3} 3$

Nr. 23 with 18 positive roots:

$1,2,3,12,13,23,1^{2} 2,123,1^{3} 2,1^{2} 23,12^{2} 3,1^{3} 23,1^{2} 2^{2} 3,1^{3} 2^{2} 3,1^{4} 2^{2} 3,1^{3} 2^{3} 3,1^{4} 2^{3} 3,1^{4} 2^{3} 3^{2}$

Nr. 24 with 19 positive roots:

$1,2,3,12,13,1^{2} 2,123,1^{3} 2,1^{2} 23,1^{4} 2,1^{3} 23,1^{4} 23,1^{3} 2^{2} 3,1^{4} 2^{2} 3,1^{5} 2^{2} 3,1^{6} 2^{2} 3,1^{6} 2^{3} 3,1^{7} 2^{3} 3,1^{7} 2^{3} 3^{2}$

Nr. 25 with 19 positive roots:

$1,2,3,12,23,1^{2} 2,123,1^{3} 2,1^{2} 23,1^{4} 2,1^{3} 23,1^{2} 2^{2} 3,1^{4} 23,1^{3} 2^{2} 3,1^{4} 2^{2} 3,1^{5} 2^{2} 3,1^{6} 2^{2} 3,1^{6} 2^{3} 3,1^{6} 2^{3} 3^{2}$

Nr. 26 with 19 positive roots:

$1,2,3,12,13,23,1^{2} 2,12^{2}, 123,1^{3} 2,1^{2} 23,12^{2} 3,1^{3} 23,1^{2} 2^{2} 3,1^{3} 2^{2} 3,1^{4} 2^{2} 3,1^{3} 2^{3} 3,1^{4} 2^{3} 3,1^{4} 2^{3} 3^{2}$

Nr. 27 with 19 positive roots:

$1,2,3,12,13,23,1^{2} 2,123,1^{3} 2,1^{2} 23,12^{2} 3,1^{3} 2^{2}, 1^{3} 23,1^{2} 2^{2} 3,1^{3} 2^{2} 3,1^{4} 2^{2} 3,1^{3} 2^{3} 3,1^{4} 2^{3} 3,1^{4} 2^{3} 3^{2}$

Nr. 28 with 19 positive roots:

$1,2,3,12,23,1^{2} 2,123,1^{3} 2,1^{2} 23,1^{3} 2^{2}, 1^{3} 23,1^{2} 2^{2} 3,1^{3} 2^{2} 3,1^{4} 2^{2} 3,1^{3} 2^{3} 3,1^{4} 2^{3} 3,1^{5} 2^{3} 3,1^{6} 2^{3} 3,1^{6} 2^{4} 3$

Nr. 29 with 20 positive roots:

$1,2,3,12,13,1^{2} 2,123,1^{3} 2,1^{2} 23,1^{4} 2,1^{3} 2^{2}, 1^{3} 23,1^{4} 23,1^{3} 2^{2} 3,1^{5} 2^{2}, 1^{4} 2^{2} 3,1^{5} 2^{2} 3,1^{6} 2^{2} 3,1^{6} 2^{3} 3,1^{7} 2^{3} 3$

Nr. 30 with 20 positive roots:

$1,2,3,12,13,1^{2} 2,123,1^{3} 2,1^{2} 23,1^{4} 2,1^{3} 2^{2}, 1^{3} 23,1^{4} 23,1^{3} 2^{2} 3,1^{4} 2^{2} 3,1^{5} 2^{2} 3,1^{6} 2^{2} 3,1^{6} 2^{3} 3,1^{7} 2^{3} 3,1^{7} 2^{3} 3^{2}$

Nr. 31 with 20 positive roots:

$1,2,3,12,13,1^{2} 2,1^{2} 3,123,1^{3} 2,1^{2} 23,1^{3} 2^{2}, 1^{3} 23,1^{2} 2^{2} 3,1^{4} 23,1^{3} 2^{2} 3,1^{4} 2^{2} 3,1^{5} 2^{2} 3,1^{4} 2^{3} 3,1^{5} 2^{3} 3,1^{6} 2^{3} 3^{2}$

Nr. 32 with 21 positive roots:

$1,2,3,12,13,1^{2} 2,123,1^{3} 2,1^{2} 23,1^{4} 2,1^{3} 2^{2}, 1^{3} 23,1^{4} 23,1^{3} 2^{2} 3,1^{5} 2^{2}, 1^{4} 2^{2} 3,1^{5} 2^{2} 3,1^{6} 2^{2} 3,1^{6} 2^{3} 3,1^{7} 2^{3} 3$, $1^{7} 2^{3} 3^{2}$ 
Nr. 33 with 21 positive roots:

$1,2,3,12,13,1^{2} 2,1^{2} 3,123,1^{3} 2,1^{2} 23,1^{3} 2^{2}, 1^{3} 23,1^{2} 2^{2} 3,1^{4} 23,1^{3} 2^{2} 3,1^{4} 2^{2} 3,1^{5} 2^{2} 3,1^{4} 2^{3} 3,1^{5} 2^{3} 3,1^{6} 2^{3} 3$, $1^{6} 2^{3} 3^{2}$

Nr. 34 with 21 positive roots:

$1,2,3,12,13,1^{2} 2,1^{2} 3,123,1^{3} 2,1^{2} 23,1^{3} 2^{2}, 1^{3} 23,1^{4} 23,1^{3} 2^{2} 3,1^{4} 2^{2} 3,1^{5} 2^{2} 3,1^{5} 2^{3} 3,1^{5} 2^{2} 3^{2}, 1^{6} 2^{3} 3,1^{6} 2^{3} 3^{2}$, $1^{7} 2^{3} 3^{2}$

Nr. 35 with 22 positive roots:

$1,2,3,12,13,1^{2} 2,1^{2} 3,123,1^{3} 2,1^{2} 23,1^{3} 2^{2}, 1^{3} 23,1^{2} 2^{2} 3,1^{4} 23,1^{3} 2^{2} 3,1^{4} 2^{2} 3,1^{5} 2^{2} 3,1^{4} 2^{3} 3,1^{5} 2^{3} 3,1^{5} 2^{2} 3^{2}$, $1^{5} 2^{3} 3^{2}, 1^{6} 2^{3} 3^{2}$

Nr. 36 with 25 positive roots:

$1,2,3,12,13,1^{2} 2,1^{2} 3,123,1^{3} 2,1^{2} 23,1^{4} 2,1^{3} 2^{2}, 1^{3} 23,1^{4} 23,1^{3} 2^{2} 3,1^{5} 2^{2}, 1^{5} 23,1^{4} 2^{2} 3,1^{5} 2^{2} 3,1^{6} 2^{2} 3,1^{7} 2^{2} 3$, $1^{6} 2^{3} 3,1^{7} 2^{3} 3,1^{8} 2^{3} 3,1^{8} 2^{3} 3^{2}$

Nr. 37 with 25 positive roots:

$1,2,3,12,13,1^{2} 2,1^{2} 3,123,1^{3} 2,1^{2} 23,1^{4} 2,1^{3} 23,1^{4} 23,1^{3} 2^{2} 3,1^{5} 23,1^{4} 2^{2} 3,1^{5} 2^{2} 3,1^{6} 2^{2} 3,1^{7} 2^{2} 3,1^{6} 2^{3} 3,1^{7} 2^{3} 3$, $1^{8} 2^{3} 3,1^{7} 2^{3} 3^{2}, 1^{8} 2^{3} 3^{2}, 1^{9} 2^{3} 3^{2}$

Nr. 38 with 25 positive roots:

$1,2,3,12,13,1^{2} 2,1^{2} 3,12^{2}, 123,1^{3} 2,1^{2} 23,12^{2} 3,1^{3} 23,1^{2} 2^{2} 3,1^{4} 23,1^{3} 2^{2} 3,1^{4} 2^{2} 3,1^{3} 2^{3} 3,1^{3} 2^{2} 3^{2}, 1^{4} 2^{3} 3$, $1^{5} 2^{3} 3,1^{4} 2^{3} 3^{2}, 1^{5} 2^{3} 3^{2}, 1^{6} 2^{3} 3^{2}, 1^{7} 2^{4} 3^{2}$

Nr. 39 with 25 positive roots:

$1,2,3,12,13,1^{2} 2,1^{2} 3,123,1^{3} 2,1^{2} 23,1^{3} 2^{2}, 1^{3} 23,1^{2} 2^{2} 3,1^{4} 23,1^{3} 2^{2} 3,1^{4} 2^{2} 3,1^{5} 2^{2} 3,1^{4} 2^{3} 3,1^{5} 2^{3} 3,1^{5} 2^{2} 3^{2}$, $1^{6} 2^{3} 3,1^{5} 2^{3} 3^{2}, 1^{6} 2^{3} 3^{2}, 1^{7} 2^{3} 3^{2}, 1^{7} 2^{4} 3^{2}$

Nr. 40 with 26 positive roots:

$1,2,3,12,13,1^{2} 2,1^{2} 3,123,1^{3} 2,1^{2} 23,1^{4} 2,1^{3} 2^{2}, 1^{3} 23,1^{4} 23,1^{3} 2^{2} 3,1^{5} 2^{2}, 1^{5} 23,1^{4} 2^{2} 3,1^{5} 2^{2} 3,1^{6} 2^{2} 3,1^{7} 2^{2} 3$, $1^{6} 2^{3} 3,1^{7} 2^{3} 3,1^{8} 2^{3} 3,1^{7} 2^{3} 3^{2}, 1^{8} 2^{3} 3^{2}$

Nr. 41 with 26 positive roots:

$1,2,3,12,13,1^{2} 2,1^{2} 3,123,1^{3} 2,1^{2} 23,1^{4} 2,1^{3} 2^{2}, 1^{3} 23,1^{4} 23,1^{3} 2^{2} 3,1^{5} 23,1^{4} 2^{2} 3,1^{5} 2^{2} 3,1^{6} 2^{2} 3,1^{7} 2^{2} 3,1^{6} 2^{3} 3$, $1^{7} 2^{3} 3,1^{8} 2^{3} 3,1^{7} 2^{3} 3^{2}, 1^{8} 2^{3} 3^{2}, 1^{9} 2^{3} 3^{2}$

Nr. 42 with 27 positive roots:

$1,2,3,12,13,1^{2} 2,1^{2} 3,123,1^{3} 2,1^{2} 23,1^{4} 2,1^{3} 2^{2}, 1^{3} 23,1^{4} 23,1^{3} 2^{2} 3,1^{5} 2^{2}, 1^{5} 23,1^{4} 2^{2} 3,1^{5} 2^{2} 3,1^{6} 2^{2} 3,1^{7} 2^{2} 3$, $1^{6} 2^{3} 3,1^{7} 2^{3} 3,1^{8} 2^{3} 3,1^{7} 2^{3} 3^{2}, 1^{8} 2^{3} 3^{2}, 1^{9} 2^{3} 3^{2}$

Nr. 43 with 27 positive roots:

$1,2,3,12,13,1^{2} 2,1^{2} 3,123,1^{3} 2,1^{2} 23,1^{4} 2,1^{3} 2^{2}, 1^{3} 23,1^{4} 23,1^{3} 2^{2} 3,1^{5} 23,1^{4} 2^{2} 3,1^{5} 2^{2} 3,1^{6} 2^{2} 3,1^{5} 2^{2} 3^{2}$, $1^{7} 2^{2} 3,1^{6} 2^{3} 3,1^{7} 2^{3} 3,1^{8} 2^{3} 3,1^{7} 2^{3} 3^{2}, 1^{8} 2^{3} 3^{2}, 1^{9} 2^{3} 3^{2}$

Nr. 44 with 27 positive roots:

$1,2,3,12,13,1^{2} 2,1^{2} 3,123,1^{3} 2,1^{2} 23,1^{4} 2,1^{3} 2^{2}, 1^{3} 23,1^{4} 23,1^{3} 2^{2} 3,1^{5} 23,1^{4} 2^{2} 3,1^{5} 2^{2} 3,1^{6} 2^{2} 3,1^{7} 2^{2} 3,1^{6} 2^{3} 3$, $1^{7} 2^{3} 3,1^{7} 2^{2} 3^{2}, 1^{8} 2^{3} 3,1^{7} 2^{3} 3^{2}, 1^{8} 2^{3} 3^{2}, 1^{9} 2^{3} 3^{2}$

Nr. 45 with 28 positive roots:

$1,2,3,12,13,1^{2} 2,1^{2} 3,123,1^{3} 2,1^{2} 23,1^{4} 2,1^{3} 2^{2}, 1^{3} 23,1^{4} 23,1^{3} 2^{2} 3,1^{5} 2^{2}, 1^{5} 23,1^{4} 2^{2} 3,1^{5} 2^{2} 3,1^{6} 2^{2} 3,1^{5} 2^{2} 3^{2}$, $1^{7} 2^{2} 3,1^{6} 2^{3} 3,1^{7} 2^{3} 3,1^{8} 2^{3} 3,1^{7} 2^{3} 3^{2}, 1^{8} 2^{3} 3^{2}, 1^{9} 2^{3} 3^{2}$

Nr. 46 with 28 positive roots:

$1,2,3,12,13,1^{2} 2,1^{2} 3,123,1^{3} 2,1^{2} 23,1^{4} 2,1^{3} 2^{2}, 1^{3} 23,1^{4} 23,1^{3} 2^{2} 3,1^{5} 23,1^{4} 2^{2} 3,1^{5} 2^{2} 3,1^{6} 2^{2} 3,1^{5} 2^{2} 3^{2}$, $1^{7} 2^{2} 3,1^{6} 2^{3} 3,1^{7} 2^{3} 3,1^{8} 2^{3} 3,1^{7} 2^{3} 3^{2}, 1^{8} 2^{3} 3^{2}, 1^{9} 2^{3} 3^{2}, 1^{9} 2^{4} 3^{2}$

Nr. 47 with 28 positive roots:

$1,2,3,12,13,1^{2} 2,1^{2} 3,123,1^{3} 2,1^{2} 23,1^{4} 2,1^{3} 2^{2}, 1^{3} 23,1^{4} 23,1^{3} 2^{2} 3,1^{5} 23,1^{4} 2^{2} 3,1^{5} 2^{2} 3,1^{6} 2^{2} 3,1^{5} 2^{2} 3^{2}$, $1^{7} 2^{2} 3,1^{6} 2^{3} 3,1^{7} 2^{3} 3,1^{8} 2^{3} 3,1^{7} 2^{3} 3^{2}, 1^{8} 2^{3} 3^{2}, 1^{9} 2^{3} 3^{2}, 1^{11} 2^{4} 3^{2}$

Nr. 48 with 29 positive roots:

$1,2,3,12,13,1^{2} 2,1^{2} 3,123,1^{3} 2,1^{2} 23,1^{4} 2,1^{3} 2^{2}, 1^{3} 23,1^{4} 23,1^{3} 2^{2} 3,1^{5} 2^{2}, 1^{5} 23,1^{4} 2^{2} 3,1^{5} 2^{2} 3,1^{6} 2^{2} 3,1^{5} 2^{2} 3^{2}$, $1^{7} 2^{2} 3,1^{6} 2^{3} 3,1^{7} 2^{3} 3,1^{7} 2^{2} 3^{2}, 1^{8} 2^{3} 3,1^{7} 2^{3} 3^{2}, 1^{8} 2^{3} 3^{2}, 1^{9} 2^{3} 3^{2}$

Nr. 49 with 29 positive roots:

$1,2,3,12,13,1^{2} 2,1^{2} 3,123,1^{3} 2,1^{2} 23,1^{4} 2,1^{3} 2^{2}, 1^{3} 23,1^{4} 23,1^{3} 2^{2} 3,1^{5} 2^{2}, 1^{5} 23,1^{4} 2^{2} 3,1^{5} 2^{2} 3,1^{6} 2^{2} 3,1^{5} 2^{2} 3^{2}$, $1^{7} 2^{2} 3,1^{6} 2^{3} 3,1^{7} 2^{3} 3,1^{8} 2^{3} 3,1^{7} 2^{3} 3^{2}, 1^{8} 2^{3} 3^{2}, 1^{9} 2^{3} 3^{2}, 1^{9} 2^{4} 3^{2}$

Nr. 50 with 29 positive roots:

$1,2,3,12,13,1^{2} 2,1^{2} 3,123,1^{3} 2,1^{2} 23,1^{4} 2,1^{3} 2^{2}, 1^{3} 23,1^{4} 23,1^{3} 2^{2} 3,1^{5} 2^{2}, 1^{5} 23,1^{4} 2^{2} 3,1^{5} 2^{2} 3,1^{6} 2^{2} 3,1^{5} 2^{2} 3^{2}$, $1^{7} 2^{2} 3,1^{6} 2^{3} 3,1^{7} 2^{3} 3,1^{8} 2^{3} 3,1^{7} 2^{3} 3^{2}, 1^{8} 2^{3} 3^{2}, 1^{9} 2^{3} 3^{2}, 1^{11} 2^{4} 3^{2}$

Nr. 51 with 30 positive roots:

$1,2,3,12,13,1^{2} 2,1^{2} 3,123,1^{3} 2,1^{2} 23,1^{4} 2,1^{3} 2^{2}, 1^{3} 23,1^{4} 23,1^{3} 2^{2} 3,1^{5} 2^{2}, 1^{5} 23,1^{4} 2^{2} 3,1^{5} 2^{2} 3,1^{6} 2^{2} 3,1^{5} 2^{2} 3^{2}$, $1^{7} 2^{2} 3,1^{6} 2^{3} 3,1^{7} 2^{3} 3,1^{7} 2^{2} 3^{2}, 1^{8} 2^{3} 3,1^{7} 2^{3} 3^{2}, 1^{8} 2^{3} 3^{2}, 1^{9} 2^{3} 3^{2}, 1^{9} 2^{4} 3^{2}$

Nr. 52 with 31 positive roots: 
$1,2,3,12,13,1^{2} 2,1^{2} 3,123,1^{3} 2,1^{2} 23,1^{4} 2,1^{3} 23,1^{5} 2,1^{4} 23,1^{6} 2,1^{5} 23,1^{4} 2^{2} 3,1^{6} 23,1^{5} 2^{2} 3,1^{7} 23,1^{6} 2^{2} 3$, ${ }_{1}^{7} 2^{2} 3,1^{8} 2^{2} 3,1^{9} 2^{2} 3,1^{10} 2^{2} 3,1^{9} 2^{3} 3,1^{10} 2^{3} 3,1^{11} 2^{3} 3,1^{10} 2^{3} 3^{2}, 1^{11} 2^{3} 3^{2}, 1^{12} 2^{3} 3^{2}$

Nr. 53 with 31 positive roots:

$1,2,3,12,13,1^{2} 2,1^{2} 3,123,1^{3} 2,1^{2} 23,1^{4} 2,1^{3} 2^{2}, 1^{3} 23,1^{4} 23,1^{3} 2^{2} 3,1^{5} 2^{2}, 1^{5} 23,1^{4} 2^{2} 3,1^{5} 2^{2} 3,1^{6} 2^{2} 3,1^{5} 2^{2} 3^{2}$, $1^{7} 2^{2} 3,1^{6} 2^{3} 3,1^{7} 2^{3} 3,1^{7} 2^{2} 3^{2}, 1^{8} 2^{3} 3,1^{7} 2^{3} 3^{2}, 1^{8} 2^{3} 3^{2}, 1^{9} 2^{3} 3^{2}, 1^{9} 2^{4} 3^{2}, 1^{11} 2^{4} 3^{2}$

Nr. 54 with 34 positive roots:

$1,2,3,12,13,1^{2} 2,1^{2} 3,123,1^{3} 2,1^{2} 23,1^{4} 2,1^{3} 2^{2}, 1^{3} 23,1^{4} 23,1^{3} 2^{2} 3,1^{5} 2^{2}, 1^{5} 23,1^{4} 2^{2} 3,1^{5} 2^{2} 3,1^{6} 2^{2} 3,1^{5} 2^{3} 3$, $1^{7} 2^{2} 3,1^{6} 2^{3} 3,1^{7} 2^{3} 3,1^{8} 2^{3} 3,1^{7} 2^{3} 3^{2}, 1^{8} 2^{4} 3,1^{8} 2^{3} 3^{2}, 1^{9} 2^{4} 3,1^{9} 2^{3} 3^{2}, 1^{9} 2^{4} 3^{2}, 1^{11} 2^{4} 3^{2}, 1^{11} 2^{5} 3^{2}, 1^{12} 2^{5} 3^{2}$

Nr. 55 with 37 positive roots:

$1,2,3,12,13,1^{2} 2,1^{2} 3,123,1^{3} 2,1^{2} 23,1^{4} 2,1^{3} 2^{2}, 1^{3} 23,1^{4} 23,1^{3} 2^{2} 3,1^{5} 2^{2}, 1^{5} 23,1^{4} 2^{2} 3,1^{5} 2^{2} 3,1^{6} 2^{2} 3,1^{5} 2^{3} 3$, $1^{7} 2^{2} 3,1^{6} 2^{3} 3,1^{7} 2^{3} 3,1^{8} 2^{3} 3,1^{7} 2^{3} 3^{2}, 1^{9} 2^{3} 3,1^{8} 2^{4} 3,1^{8} 2^{3} 3^{2}, 1^{9} 2^{4} 3,1^{9} 2^{3} 3^{2}, 1^{10} 2^{4} 3,1^{9} 2^{4} 3^{2}, 1^{11} 2^{4} 3^{2}, 1^{11} 2^{5} 3^{2}$, $1^{12} 2^{5} 3^{2}, 1^{13} 2^{5} 3^{2}$

\section{ACKNOWLEDGEMENT}

The authors would like to thank B. Mühlherr for pointing out to us the importance of the number 37 for simplicial arrangements in the real projective plane. The authors are also thankful to the referee for helping us clarify some ambiguities.

\section{REFERENCES}

[Bou68] N. Bourbaki, Groupes et algèbres de Lie, ch.4, 5 et 6, Éléments de mathématique, Hermann, Paris, 1968. MR0240238 (39:1590)

[CH09a] M. Cuntz and I. Heckenberger, Reflection groupoids of rank two and cluster algebras of type A, Preprint arXiv:0911.3051v1 (2009), 18 pp.

[CH09b] _ Weyl groupoids of rank two and continued fractions, Algebra and Number Theory 3 (2009), 317-340. MR 2525553

[CH09c] - Weyl groupoids with at most three objects, J. Pure Appl. Algebra 213 (2009), 1112-1128. MR2498801 (2010b:20100)

[Grü09] B. Grünbaum, A catalogue of simplicial arrangements in the real projective plane, Ars Math. Contemp. 2 (2009), 1-25. MR2485643(2010d:51033)

[Hec05] I. Heckenberger, Classification of arithmetic root systems of rank 3, Actas del "XVI Coloquio Latinoamericano de Álgebra" (Colonia, Uruguay, 2005), 2005, pp. 227-252. MR 2500361

[Hec06] - The Weyl groupoid of a Nichols algebra of diagonal type, Invent. Math. 164 (2006), 175-188. MR2207786 (2007e:16047)

[Hec08] - Rank 2 Nichols algebras with finite arithmetic root system, Algebr. Represent. Theory 11 (2008), 115-132. MR.2379892 (2009a:16080)

[HY08] I. Heckenberger and H. Yamane, A generalization of Coxeter groups, root systems, and Matsumoto's theorem, Math. Z. 259 (2008), 255-276.

[Kac77] V.G. Kac, Lie superalgebras, Adv. Math. 26 (1977), 8-96. MR0486011(58:5803)

[Kac90] , Infinite dimensional Lie algebras, Cambridge Univ. Press, 1990. MR1104219 (92k:17038)

[Mel41] E. Melchior, Über Vielseite der projektiven Ebene, Deutsche Mathematik 5 (1941), 461475. MR0004476 (3:13c)

[tD91] T. tom Dieck, Topologie, de Gruyter Lehrbuch, Walter de Gruyter \& Co., Berlin, 1991. MR 1150244 (93f:55001)

Fachbereich Mathematik, Universität Kaiserslautern, Postfach 3049, D-67653 Kaiserslautern, Germany

E-mail address: cuntz@mathematik.uni-kl.de

Fachbereich Mathematik und Informatik, Philipps-Universität Marburg, HansMeerwein-Strasse, D-35032 Marburg, Germany

E-mail address: heckenberger@mathematik.uni-marburg.de 